\title{
Extremals for the Singular Moser-Trudinger Inequality via n-Harmonic Transplantation
}

July 31,2020

\author{
Gyula Csató $^{1}$, Prosenjit Roy ${ }^{2}$ and Van Hoang NGuyen ${ }^{3}$ \\ 1. Universitat Politècnica de Catalunya and member of BGSMath Barcelona, Spain, \\ supported by Fondecyt grant no. 11150017, by the María de Maeztu Grant MDM-2014-0445 \\ and by MINECO grant MTM2017-84214-C2-1-P. \\ 2. Indian Institute of Teechnology, Kanpur, India \\ 3. Institute of Mathematics, Vietnam Academy of Science and Technology, \\ Hanoi, Vietnam
}

\begin{abstract}
The Moser-Trudinger embedding has been generalized in [Adimurthi A.; Sandeep K., A singular Moser-Trudinger embedding and its applications, NoDEA Nonlinear Differential Equations Appl., 13 (2007), no. 5-6, 585-603] to the following weighted version: if $\Omega \subset \mathbb{R}^{n}$ is bounded, $\omega_{n-1}$ is the $\mathcal{H}^{n-1}$ measure of the unit sphere, then for $\alpha>0$ and $\beta \in[0, n)$,

$$
\sup _{u \in \mathcal{B}_{1}} \int_{\Omega} \frac{e^{\alpha|u|^{n /(n-1)}}}{|x|^{\beta}} \leq C \Leftrightarrow \frac{\alpha}{\alpha_{n}}+\frac{\beta}{n} \leq 1,
$$

where $\alpha_{n}=n \omega_{n-1}^{1 /(n-1)}$ and $\mathcal{B}_{1}=\left\{\left.u \in W_{0}^{1, n}(\Omega)\left|\int_{\Omega}\right| \nabla u\right|^{n} \leq 1\right\}$. We prove that the supremum is attained on any domain $\Omega$. The paper also fills in the gaps in the proof of [Lin K.C., Extremal functions for Moser's inequality, Trans. of. Am. Math. Soc., 384 (1996), 2663-2671], which deals with the case $\beta=0$.
\end{abstract}

\section{Introduction}

Let $\Omega \subset \mathbb{R}^{n}$ be a bounded open smooth set. The Moser-Trudinger imbedding, which is due to Trudinger [39] and in its sharp form to Moser [32], states that the following supremum is finite

$$
\sup _{\substack{v \in W_{0}^{1, n}(\Omega) \\\|\nabla v\|_{L^{n} \leq 1} \leq}} \int_{\Omega}\left(e^{n \omega_{n-1}^{1 /(n-1)} v^{\frac{n}{n-1}}}-1\right)<\infty .
$$

First it has been shown by Carleson-Chang $[8$ that the supremum is actually attained, if $\Omega$ is a ball. In [36], Struwe proved for $n=2$ that the result remains true if $\Omega$ is close to a ball. Using the

2010 Mathematics Subject Classification. Primary 26D10 (46E35, 52A40)

Key words and phrases. Moser Trudinger inequality, extremal function. 
harmonic transplantation method Flucher 17] generalized this result to arbitrary bounded domains in $\mathbb{R}^{2}$. If $n=2$ and $\Omega$ is the unit disk some new proofs have been obtained of the Moser-Trudinger inequality, respectively of the Carleson-Chang's result in Mancini-Martinazzi 28. Other results dealing with critical points and extremal functions of the Moser-Trudinger inequality, or its sharper version, have also been obtained in Malchiodi-Martinazzi [27] (for $\Omega$ equal the unit disk), AdimurthiDruet [1 and Yang [40, 41] (for a version on manifolds) and [42. These results use blow up analysis and are all restricted to 2 dimensions. See also Adimurthi-Tintarev [3], Mancini-Sandeep [29, 30], [31, [37] and Yang [43] and the references in these papers for other recent developments on the subject. However, the only result dealing with the extremal functions in higher dimensions $n \geq 3$ and more general bounded domains than balls, is by Lin [25]. His proof is however rather sketchy in some parts and we believe he missed several important and nontrivial points. In particular the entire construction of Section 7 in this paper is necessary in the case $\beta=0$ too, as well as the use of all the nontrivial results on the $n$-Laplacian we summarized in the Appendix. Setting $\beta=0$ in this present paper we implicitly give rigorous proofs to all of Lins claims.

The Moser-Trudinger embedding has been generalized by Adimurthi-Sandeep 2] to a singular version, which reads as the following: If $\alpha>0$ and $\beta \in[0, n)$ is such that

$$
\frac{\alpha}{\alpha_{n}}+\frac{\beta}{n} \leq 1, \quad \text { where } \alpha_{n}=n \omega_{n-1}^{1 /(n-1)}
$$

$\omega_{n-1}$ is the $\mathcal{H}^{n-1}$ measure of the unit sphere, then the following supremum is finite

$$
\sup _{\substack{v \in W_{0}^{1, n}(\Omega) \\\|\nabla v\|_{L^{n}} \leq 1}} \int_{\Omega} \frac{e^{\alpha|v|^{\frac{n}{n-1}}}-1}{|x|^{\beta}}<\infty .
$$

In the case $n=2$ it was proven first in Csató-Roy [11, 12] that the supremum is attained. Afterwards, using blow-up analyis, the same result and some refinements have also been obtained by Yang and Zhu 44. Their proof is based on classification theorems in 2 dimensions by Chen and Li [13, 14. Recently, also in 2 dimensions, a third proof was given by Lula-Mancini 26, also depending on [13]. We point out that there is no available Chen-Li type classification result in higher dimensions. The aim of this paper is to generalize [11 to higher dimensions and we prove the following theorem.

Theorem 1 Let $n \geq 2$ and $\Omega \subset \mathbb{R}^{n}$ be a bounded open smooth set. Let $\alpha>0$ and $\beta \in[0, n)$ be such that (10) is satisfied. Then there exists $u \in W_{0}^{1, n}(\Omega)$ such that $\|\nabla u\|_{L^{n}} \leq 1, u \geq 0$ and

$$
\sup _{\substack{v \in W_{0}^{1, n}(\Omega) \\\|\nabla v\|_{L^{n} \leq 1}}} \int_{\Omega} \frac{e^{\alpha|v|^{\frac{n}{n-1}}}-1}{|y|^{\beta}} d y=\int_{\Omega} \frac{e^{\alpha u^{\frac{n}{n-1}}}-1}{|y|^{\beta}} d y .
$$

It is an immediate consequence of this theorem, that $u \in W_{0}^{1, n}$ gives a weak solution, for some $\mu>0$, to the following semilinear elliptic eqaution involving the $n$-Laplacian

$$
\left\{\begin{aligned}
-\Delta_{n} u=\mu u^{\frac{1}{n-1}} e^{\alpha u^{\frac{n}{n-1}}} \frac{1}{|y|^{\beta}} & \text { in } \Omega \\
u \geq 0 & \text { in } \Omega \\
u=0 & \text { on } \partial \Omega
\end{aligned}\right.
$$


satisfying moreover $\int_{\Omega}|\nabla u|^{n}=1$.

The proof of Theorem 1 follows the ideas of Flucher [17, respectively Lin 25] and we have given an overview of the method in Csató-Roy [1], which we will not repeat here. The case $0 \notin \bar{\Omega}$ is rather elementery and exactly as in the 2-dimensional case, see [11, and the main difficulty is the case $0 \in \Omega$. The proof of Theorem 1 roughly speaking, consists in reducing the problem to a kind of isoperimetric problem with density involving the $n$-Green's function. The $n$-Green's function $G_{\Omega, 0}$ of a general domain $\Omega$ containing the origin has the form

$$
G_{\Omega, 0}(y)=-\frac{1}{\omega_{n-1}^{1 /(n-1)}} \log |y|-H(y),
$$

where $H$ is the regular part. The conformal incenter at 0 is then defined by

$$
I_{\Omega}(0)=e^{-\omega_{n-1}^{1 /(n-1)} H(0)} .
$$

This is usually called the conformal inradius at 0 , see Flucher [18 and we have adopted the name conformal incenter at 0 in [11 mistakenly. However, in this paper, we will stick to this name for consistency with [11, 12]. The isoperimetric problem to which the question of the existence of extremal function is reduced is the following inequality:

$$
\omega_{n-1}^{\frac{n}{n-1}} I_{\Omega}^{n-\beta}(0) \leq \int_{\partial \Omega} \frac{1}{|y|^{\beta}\left|\nabla G_{\Omega, 0}(y)\right|} d \mathcal{H}^{n-1}(y) \quad \text { for any } \Omega \text { with } 0 \in \Omega .
$$

There is equality for balls cetered at the origin. Let us now point out the two main differences and difficulties compared to the 2-dimensional case:

1. The reduction of the problem to (3) uses the $n$-harmonic transplantation. This uses existence, regularity and other properties of th $n$-Laplace equation, which is a linear equation only if $n=2$. Solutions to the $n$-Laplace equation have moreover weaker regularity properties if $n>2$. The difficulties are more of technical type and are mostly relevant in Section 7

2. If $n=2$ then (3) was proven by the following three inequalities (where $R_{\Omega}$ is the radius of $\Omega^{*}$, i.e. $\left.\pi R_{\Omega}^{2}=|\Omega|\right)$

$$
\begin{aligned}
\omega_{1}^{2} I_{\Omega}^{2-\beta}(0) \underset{(\mathrm{a})}{\leq} \omega_{1}^{2} R_{\Omega}^{2-\beta}=\omega_{1}^{2}\left(\frac{|\Omega|}{\pi}\right)^{\frac{2-\beta}{2}} \underset{(\mathrm{b})}{\leq}\left(\int_{\partial \Omega} \frac{1}{|y|^{\beta / 2}} d \mathcal{H}^{1}(y)\right)^{2} \\
=\left(\int_{\partial \Omega} \frac{\sqrt{|\nabla G|}}{\sqrt{|y|^{\beta}|\nabla G|}} d \mathcal{H}^{1}(y)\right)^{2} \leq \int_{\partial \Omega} \frac{1}{|y|^{\beta}|\nabla G|} d \mathcal{H}^{1}(y) .
\end{aligned}
$$

The estimate (a), i.e. $I_{\Omega} \leq R_{\Omega}$ is standard, (b) is a weighted isoperimetric inequality and (c) is just Hölder inequality and the elementery property $\int_{\partial \Omega}|\nabla G|=1$. This is the method followed by Flucher [17, Csató-Roy [11]. If $\beta=0$, then the same steps work in any dimension, just by using the classical isoperimetric inequality and this is what was used by Lin 25 . If $\beta \neq 0$, then the corresponding weighted isoperimetric inequality was proven by Csató 9 if $n=2$. However, a higher dimensional version of the appropriate weighted isoperimetric inequality fails, see Csató [10] Theorem 9 (ii) and Example 11. Example 11 shows precisely that such an isoperimetric inequality even fails for any ball not centered at the origin. Moreover, if $n \geq 3$ both estimates (a) and (c) are too generous to prove (3), as numerical evidence suggests. So a completely new method had to be 
developed to prove the higher dimensional case, without using any of the estimates (a)-(c).This new method relies on a careful analysis of the properties of $G_{\Omega, 0}$ and on a different weighted isoperimetric inequality by Alvino, Brock, Chiacchio, Mercaldo and Posteraro 4 Theorem 1.1 (ii), which reads as.

$$
\int_{\Omega}|y|^{-\beta} d y \leq C\left(\int_{\partial \Omega}|y|^{-\frac{n-1}{n} \beta} d \mathcal{H}^{n-1}(y)\right)^{\frac{n}{n-1}} \quad \text { for any smooth set } \Omega \subset \mathbb{R}^{n},
$$

where $C$ is such that equality holds for balls centered at the origin. The proof of (3) is contained in Section 3

\section{Notations and Definitions}

Throughout this paper $n \geq 2$ is an integer and $\Omega \subset \mathbb{R}^{n}$ will denote a bounded open set with smooth boundary $\partial \Omega$. Its $n$-dimensional Lebesgue measure is written as $|\Omega|$. The $(n-1)$-dimensional Hausdorff measure is denoted by $\mathcal{H}^{n-1}$. Balls with radius $R$ and center at $x$ are written $B_{R}(x) \subset \mathbb{R}^{n}$; if $x=0$, we simply write $B_{R}$. The space $W^{1, n}(\Omega)$ denotes the usual Sobolev space of functions and $W_{0}^{1, n}(\Omega)$ those Sobolev functions with vanishing trace on the boundary. Throughout this paper $\alpha, \beta \in \mathbb{R}$ are two constants satisfying $\alpha>0, \beta \in[0, n)$ and

$$
\frac{\alpha}{\alpha_{n}}+\frac{\beta}{n} \leq 1, \quad \text { where } \alpha_{n}=n \omega_{n-1}^{1 /(n-1)}
$$

where $\omega_{n-1}$ is the $\mathcal{H}^{n-1}$ measure of the unit sphere.

- We define the the funtctionals $F_{\Omega}, J_{\Omega}: W_{0}^{1, n}(\Omega) \rightarrow \mathbb{R}$ by

$$
\begin{aligned}
& F_{\Omega}(u)=\int_{\Omega} \frac{e^{\alpha|u|^{\frac{n}{n-1}}}-1}{|x|^{\beta}} d x, \\
& J_{\Omega}(u)=\int_{\Omega}\left(e^{\alpha_{n}|u|^{\frac{n}{n-1}}}-1\right) d x .
\end{aligned}
$$

- We say that a sequence $\left\{u_{i}\right\} \subset W_{0}^{1, n}(\Omega)$ concentrates at $x \in \bar{\Omega}$ if

$$
\lim _{i \rightarrow \infty}\left\|\nabla u_{i}\right\|_{L^{n}}=1 \quad \text { and } \quad \forall \epsilon>0 \quad \lim _{i \rightarrow \infty} \int_{\Omega \backslash B_{\epsilon}(x)}\left|\nabla u_{i}\right|^{n}=0 .
$$

This definition is equivalent to the convergence $\left|\nabla u_{i}\right|^{n} d x \rightarrow \delta_{x}$ weakly in measure, where $\delta_{x}$ is the Dirac measure at $x$. We will use the following well known property of concentrating sequences: if $\left\{u_{i}\right\}$ concentrates, then $u_{i} \rightarrow 0$ in $W^{1, n}(\Omega)$. In particular

$$
u_{i} \rightarrow 0 \quad \text { in } L^{q}(\Omega) \quad \text { for all } q<\infty .
$$

- We define the sets

$$
W_{0, \text { rad }}^{1, n}\left(B_{1}\right)=\left\{u \in W_{0}^{1, n}\left(B_{1}\right) \mid u \text { is radial }\right\}
$$


and analogously $C_{c, \text { rad }}^{\infty}\left(B_{1}\right)$ is the set of radially symmetric smooth functions with compact support in $B_{1}$. By abuse of notation we will usually write $u(x)=u(|x|)$ for $u \in W_{0, \text { rad }}^{1, n}\left(B_{1}\right)$. The space $C_{c, \text { rad }}^{\infty}\left(B_{1}\right)$ is dense in $W_{0, \text { rad }}^{1, n}\left(B_{1}\right)$ in the $W^{1, n}$ norm. If in addition $u$ is radially decreasing we write $u \in W_{0, \text { rad } \searrow}^{1, n}\left(B_{1}\right)$, respectively $u \in C_{c, \text { rad } \searrow}^{\infty}\left(B_{1}\right)$.

- Define

$$
\mathcal{B}_{1}(\Omega)=\left\{u \in W_{0}^{1, n}(\Omega) \mid\|\nabla u\|_{L^{n}} \leq 1\right\} .
$$

- Finally we define

$$
F_{\Omega}^{\text {sup }}=\sup _{u \in \mathcal{B}_{1}(\Omega)} F_{\Omega}(u)
$$

$J_{\Omega}^{\text {sup }}$ is defined in an analogous way, replacing $F$ by $J$. If $x \in \bar{\Omega}$ and the supremum is taken only over concentrating sequences, we write $F_{\Omega}^{\delta}(x)$, more precisely

$$
F_{\Omega}^{\delta}(x)=\sup \left\{\limsup _{i \rightarrow \infty} F_{\Omega}\left(u_{i}\right) \mid \quad\left\{u_{i}\right\} \subset \mathcal{B}_{1}(\Omega) \text { concentrates at } x\right\} .
$$

We define in an analogous way $J_{\Omega}^{\delta}(x)$. If $\Omega=B_{1}$, then we define

$$
\begin{gathered}
F_{B_{1}, \text { rad } \searrow}^{\text {sup }}=\sup _{u \in W_{0, \text { rad } \searrow}^{1, n}\left(B_{1}\right) \cap \mathcal{B}_{1}\left(B_{1}\right)} F_{B_{1}}(u), \\
F_{B_{1}, \text { rad } \searrow}^{\delta}(0)=\sup \left\{\limsup _{i \rightarrow \infty} F_{B_{1}}\left(u_{i}\right) \mid\left\{u_{i}\right\} \subset W_{0, \text { rad } \searrow}^{1, n}\left(B_{1}\right) \cap \mathcal{B}_{1}\left(B_{1}\right) \text { concentrates at } 0\right\} .
\end{gathered}
$$

We define $J_{B_{1}, \text { rad }}^{\text {sup }}$ and $J_{B_{1}, \text { rad } \searrow}^{\delta}(0)$ in an analogous way.

- If $\Omega \subset \mathbb{R}^{n}$ then $\Omega^{*}$ is its symmetric rearrangement, that is $\Omega^{*}=B_{R}(0)$, where $|\Omega|=$ $R^{n} \omega_{n-1} / n$. If $u \in W_{0}^{1, n}(\Omega)$, then $u^{*} \in W_{0, \text { rad } \backslash}^{1, n}\left(B_{R}(0)\right)$ will denote the Schwarz symmetrization of $u$. For basic propertis of the Schwarz symmetrization we refer to Kesavan 20, Chapters 1 and 2, which we will use throughout. In particular we will use frequently and without further comment that if $u \in W_{0}^{1,2}(\Omega)$, then $u^{*}$ satisfies

$$
F_{B_{R}}(u) \leq F_{B_{R}}\left(u^{*}\right) \quad \text { if } \Omega=B_{R} \quad \text { and } \quad\left\|\nabla u^{*}\right\|_{L^{n}\left(B_{R}\right)} \leq\|\nabla u\|_{L^{n}(\Omega)} .
$$

We will additionally need, as in Flucher [17, a slight modification of the Hardy-Littlewood, respectively Pólya-Szegö theorem, stated in the next proposition.

Proposition 2 (i) Lef $f \in L^{p}(\Omega)$ and $g \in L^{q}(\Omega)$, where $1 / p+1 / q=1$. Then for any $a \in \mathbb{R}$

$$
\int_{\{f \geq a\}} f g \leq \int_{\left\{f^{*} \geq a\right\}} f^{*} g^{*} .
$$

(ii) Let $u \in W_{0}^{1, n}(\Omega)$ such that $u \geq 0$. Then for any $t \in(0, \infty)$

$$
\int_{\left\{u^{*} \leq t\right\}}\left|\nabla u^{*}\right|^{n} \leq \int_{\{u \leq t\}}|\nabla u|^{n} \quad \text { and } \quad \int_{\left\{u^{*} \geq t\right\}}\left|\nabla u^{*}\right|^{n} \leq \int_{\{u \geq t\}}|\nabla u|^{n} .
$$




\section{3 -Green's function}

If $x \in \Omega$, then $G_{\Omega, x}$ will denote the $n$-Green's function of $\Omega$ with singularity at $x$. It is the unique function defined on $\Omega \backslash\{x\}$ such that the principal value of the integral

$$
\int_{\Omega}\left|\nabla G_{\Omega, x}(y)\right|^{n-2}\left\langle\nabla G_{\Omega, x}(y) ; \nabla \varphi(y)\right\rangle d y=\varphi(x) \quad \text { for all } \varphi \in C_{c}^{1}(\Omega)
$$

and $G_{\Omega, x}=0$ on $\partial \Omega$. It can always be decomposed in the form

$$
G_{\Omega, x}(y)=-\frac{1}{\omega_{n-1}^{1 /(n-1)}} \log (|x-y|)-H_{\Omega, x}(y), \quad y \in \Omega \backslash\{x\},
$$

where $H_{\Omega, x}$ is a continuous function on $\bar{\Omega}$ and is $C_{l o c}^{1, \alpha}$ in $\Omega \backslash\{x\}$. This result is due to 21] (see therein Theorem stated in (0.10) and (0.11) or Theorem 2.1 and in particular Remark 1.4). Another useful reference on the $n$-Green's function is 45.

The conformal incenter $I_{\Omega}(x)$ of $\Omega$ at $x$ is defined by

$$
I_{\Omega}(x)=e^{-\omega_{n-1}^{1 /(n-1)} H_{\Omega, x}(x)} .
$$

Before stating the next proposition we need the following definition.

Definition 3 We say that a sequence of sets $\left\{A_{i}\right\} \subset \mathbb{R}^{n}$ are approximately small balls at $x \in \mathbb{R}^{n}$ (of radius $\tau_{i}$ ) as $i \rightarrow \infty$ if there exists sequences $\tau_{i}, \sigma_{i}>0$ such that $\lim _{i \rightarrow \infty} \tau_{i}=0$,

$$
\lim _{i \rightarrow \infty} \frac{\sigma_{i}}{\tau_{i}}=0
$$

and

$$
B_{\tau_{i}-\sigma_{i}}(x) \subset A_{i} \subset B_{\tau_{i}+\sigma_{i}}(x) \quad \text { for all } i \text { big enough. }
$$

We will need the following properties of the $n$-Green's function. For what follows it is convenient to abbreviate, for $\beta \in[0, n)$

$$
\alpha_{n, \beta}=(n-\beta) \omega_{n-1}^{1 /(n-1)} .
$$

Proposition 4 Let $x \in \Omega$. Then $G_{\Omega, x}$ and $I_{\Omega}(x)$ have the following properties:

(a) For every $t \in[0, \infty)$

$$
\int_{\left\{G_{\Omega, x}<t\right\}}\left|\nabla G_{\Omega, x}(y)\right|^{n} d y=t .
$$

(b) For every $t \in[0, \infty)$

$$
\int_{\left\{G_{\Omega, x}=t\right\}}\left|\nabla G_{\Omega, x}(y)\right|^{n-1} d \mathcal{H}^{n-1}(y)=1 .
$$

(c)

$$
\lim _{t \rightarrow \infty} \frac{n\left|\left\{G_{\Omega, x}>t\right\}\right|}{\omega_{n-1} e^{-n \omega_{n-1}^{1 /(n-1)} t}}=\left(I_{\Omega}(x)\right)^{n} .
$$


(d) If $B_{R}=\Omega^{*}$ is the symmetrized domain, then for any $x \in \Omega$

$$
I_{\Omega}(x) \leq I_{B_{R}}(0)=R
$$

(e) If $t_{i} \geq 0$ is a given sequence such that $t_{i} \rightarrow \infty$, then the sets $\left\{G_{\Omega, x}>t_{i}\right\}$ are approximately small balls at $x$ of radius $\tau_{i}$

$$
\tau_{i}=I_{\Omega}(x) e^{-\omega_{n-1}^{1 /(n-1)} t_{i}}
$$

In particular

$$
\lim _{t \rightarrow \infty} \frac{n-\beta}{\omega_{n-1} e^{-\alpha_{n, \beta} t}} \int_{\left\{G_{\Omega, x}>t\right\}}|y-x|^{-\beta}=I_{\Omega}(x)^{n-\beta} .
$$

Proof In all statements we can assume without loss of generality that $x=0 \in \Omega$ and abbreviate $G_{\Omega, x}=G, H_{\Omega, x}=H$.

(a) By approximation (5) holds also for any $\varphi \in W_{0}^{1, \infty}(\Omega)$. Thus (a) follows by taking $\varphi(y)=$ $\min \{t, G(y)\}$.

(b) Observe that by (a) and the coarea formula

$$
t=\int_{\{G<t\}}|\nabla G(y)|^{n-1}|\nabla G(y)| d y=\int_{0}^{t}\left(\int_{\{G=s\}}|\nabla G(y)|^{n-1} d \mathcal{H}^{n-1}(y)\right) d s .
$$

Hence, (b) follows from (a) by derivation.

(c) Step 1. Write $G$ as

$$
G(y)=-\frac{1}{\omega_{n-1}^{1 /(n-1)}} \log (|y|)-H(0)+H(0)-H(y)=-\frac{1}{\omega_{n-1}^{1 /(n-1)}} \log \left(\frac{|y|}{I_{\Omega}(0)}\right)+H(0)-H(y)
$$

Let $\|H\|_{\infty}=\sup \{|H(y)|: y \in \Omega\}$ and define

$$
S(t)=\left\{y \in \Omega:|y| \leq I_{\Omega}(0) e^{-\omega_{n-1}^{1 /(n-1)}\left(t-2\|H\|_{\infty}\right)}\right\},
$$

and set

$$
m(t)=\max _{y \in S(t)}|H(y)-H(0)|
$$

By the continuity of $H$ it holds that

$$
\lim _{t \rightarrow \infty} m(t)=0 .
$$

We now define also the sets

$$
\begin{aligned}
& P(t)=\left\{y \in \Omega:|y|<I_{\Omega}(0) e^{-\omega_{n-1}^{1 /(n-1)}(t+m(t))}\right\} \\
& Q(t)=\left\{y \in \Omega:|y|<I_{\Omega}(0) e^{-\omega_{n-1}^{1 /(n-1)}(t-m(t))}\right\},
\end{aligned}
$$

and claim that for all $t \geq 2\|H\|_{\infty}$

$$
P(t) \subset\{G>t\} \subset Q(t) .
$$


Let $y \in P(t)$, then also $y \in S(t)$. So using ([6), (8) and finally the definition of $P(t)$ we get

$$
G(y) \geq-\frac{1}{\omega_{n-1}^{1 /(n-1)}} \log \left(\frac{|y|}{I_{\Omega}(0)}\right)-m(t)=-\frac{1}{\omega_{n-1}^{1 /(n-1)}} \log \left(\frac{|y|}{I_{\Omega}(0)}\right)-(m(t)+t)+t>t .
$$

This shows $P(t) \subset\{G>t\}$. If $y \in\{G>t\}$, then using again (6) we obtain

$$
|y|<I_{\Omega}(0) e^{-\omega_{n-1}^{1 /(n-1)}(t-H(0)+H(y))} .
$$

As $t-H(0)+H(y) \geq t-2\|H\|_{\infty}$ it holds that $y \in S(t)$ and hence $|H(0)-H(y)| \leq m(t)$. This implies that $y \in Q(t)$ and proves the claim (10). It now follows from (10) that

$$
\frac{\omega_{n-1}}{n} I_{\Omega}(0)^{n} e^{-\omega_{n-1}^{1 /(n-1)}(t+m(t)) n} \leq|\{G>t\}| \leq \frac{\omega_{n-1}}{n} I_{\Omega}(0)^{n} e^{-\omega_{n-1}^{1 /(n-1)}(t-m(t)) n}
$$

Using (9) proves (c).

(d) We refer to Flucher [18 Lemma 8.2 page 64, or Lin 25] Lemma 2.

(e) is deduced from (10) in the following way: write

$$
I_{\Omega}(0) e^{-\omega_{n-1}^{1 /(n-1)}(t+m(t))}=\tau(t)-\underline{\sigma}(t), \quad I_{\Omega}(0) e^{-\omega_{n-1}^{1 /(n-1)}(t-m(t))}=\tau(t)+\bar{\sigma}(t),
$$

where

$$
\begin{aligned}
& \tau(t)=I_{\Omega}(0) e^{-\omega_{n-1}^{1 /(n-1)} t}, \quad \underline{\sigma}(t)=I_{\Omega}(0) e^{-\omega_{n-1}^{1 /(n-1)} t}\left(1-e^{-\omega_{n-1}^{1 /(n-1)} m(t)}\right) \\
& \text { and } \quad \bar{\sigma}(t)=I_{\Omega}(0) e^{-\omega_{n-1}^{1 /(n-1)} t}\left(e^{\omega_{n-1}^{1 /(n-1)} m(t)}-1\right)
\end{aligned}
$$

Using (9)

$$
\lim _{t \rightarrow \infty} \frac{\underline{\sigma}(t)}{\tau(t)}=\lim _{t \rightarrow \infty} \frac{\bar{\sigma}(t)}{\tau(t)}=0,
$$

and the first statement follows by setting $\tau_{i}=\tau\left(t_{i}\right), \sigma_{i}=\max \left\{\underline{\sigma}\left(t_{i}\right), \bar{\sigma}\left(t_{i}\right)\right\}$. The second statment follows from

$$
\int_{B_{\tau(t)-\sigma(t)}}|y|^{-\beta} d y \leq \int_{\{G>t\}}|y|^{-\beta} d y \leq \int_{B_{\tau(t)+\sigma(t)}}|y|^{-\beta} d y,
$$

calculating explicitly the first and last integral, and using the first statement of (e).

We will need the following result.

Lemma 5 Let $\Omega$ be any smooth bounded domain of $\mathbb{R}^{n}$ and $x \in \Omega$. Suppose $\beta \in[0, n)$, then it holds

$$
\int_{\Omega}|y-x|^{-\beta} d y \geq \frac{\omega_{n-1}}{n-\beta} I_{\Omega}(x)^{n-\beta} .
$$

Proof It is enough to prove (11) for $x=0$ and assume $0 \in \Omega$. We start the proof by recalling a sharp weighted isoperimetric inequality from [4] Theorem 1.1 (ii).

$$
\int_{A}|y|^{-\beta} d y \leq \frac{1}{\alpha_{n, \beta}}\left(\int_{\partial A}|y|^{-\frac{n-1}{n} \beta} d \mathcal{H}^{n-1}(y)\right)^{\frac{n}{n-1}} \quad \text { for any smooth set } A \subset \mathbb{R}^{n} .
$$


Applying (12) to the set $\left\{G_{\Omega, 0}>t\right\}$ and using Hölder inequality and Proposition廿(b), we have

$$
\begin{aligned}
\int_{\left\{G_{\Omega, 0}>t\right\}}|y|^{-\beta} d y & \leq \frac{1}{\alpha_{n, \beta}}\left(\int_{\left\{G_{\Omega, 0}=t\right\}}\left|\nabla G_{\Omega, 0}(y)\right|^{\frac{n-1}{n}} \frac{|y|^{-\frac{n-1}{n} \beta}}{\left|\nabla G_{\Omega, 0}(y)\right|^{\frac{n-1}{n}}} d \mathcal{H}^{n-1}(y)\right)^{\frac{n}{n-1}} \\
& \leq \frac{1}{\alpha_{n, \beta}}\left(\int_{\left\{G_{\Omega, 0}=t\right\}}\left|\nabla G_{\Omega, 0}(y)\right|^{n-1} d \mathcal{H}^{n-1}(y)\right)^{\frac{1}{n-1}}\left(\int_{\left\{G_{\Omega, 0}=t\right\}} \frac{|y|^{-\beta}}{\left|\nabla G_{\Omega, 0}(y)\right|} d \mathcal{H}^{n-1}(y)\right) \\
& =\frac{1}{\alpha_{n, \beta}} \int_{\left\{G_{\Omega, 0}=t\right\}} \frac{|y|^{-\beta}}{\left|\nabla G_{\Omega, 0}(y)\right|} d \mathcal{H}^{n-1}(y)
\end{aligned}
$$

By co-area formula, we have

$$
\int_{\left\{G_{\Omega, 0}>t\right\}}|y|^{-\beta} d y=\int_{t}^{\infty} \int_{\left\{G_{\Omega, 0}=s\right\}} \frac{|y|^{-\beta}}{\left|\nabla G_{\Omega, 0}(y)\right|} d \mathcal{H}^{n-1}(y) d s .
$$

Whence (13) can be rewritten as

$$
\frac{d}{d t}\left(e^{\alpha_{n, \beta} t} \int_{\left\{G_{\Omega, 0}>t\right\}}|y|^{-\beta} d y\right) \leq 0 .
$$

In other words, $t \rightarrow e^{\alpha_{n, \beta} t} \int_{\left\{G_{\Omega, 0}>t\right\}}|y|^{-\beta} d y$ is a non-increasing function. Using Proposition 4 (e) we get

as wanted.

$$
\int_{\Omega}|y|^{-\beta} d y \geq \lim _{t \rightarrow \infty} e^{\alpha_{n, \beta} t} \int_{\left\{G_{\Omega, 0}>t\right\}}|y|^{-\beta} d x=\frac{\omega_{n-1}}{n-\beta} I_{\Omega}(0)^{n-\beta}
$$

The following proposition and its corollary are the main results of this section. We have included also the case $\beta=n$, although this is not needed for the application to the singular Moser-Trudinger functional.

Proposition 6 Let $\Omega \subset \mathbb{R}^{n}$ be any smooth bounded set and $x \in \Omega$. Suppose $\beta \in[0, n]$, then it holds that

$$
\omega_{n-1}^{n /(n-1)} I_{\Omega}(x)^{n-\beta} \leq \int_{\partial \Omega} \frac{|x-y|^{-\beta}}{\left|\nabla G_{\Omega, x}(y)\right|} d \mathcal{H}^{n-1}(y)
$$

Proof Let us first assume that $\beta \in[0, n)$. We can assume without loss of generality that $x=0 \in \Omega$ and write again $G=G_{\Omega, x}$. Applying (13) to $t=0$, we have

$$
\int_{\Omega}|y|^{-\beta} d y \leq \frac{1}{\alpha_{n, \beta}} \int_{\partial \Omega} \frac{|y|^{-\beta}}{|\nabla G(y)|} d \mathcal{H}^{n-1}(y) .
$$

It then follows from (11) that

$$
\omega_{n-1}^{n /(n-1)} I_{\Omega}(0)^{n-\beta}=\alpha_{n, \beta} \frac{\omega_{n-1}}{n-\beta} I_{\Omega}(0)^{n-\beta} \leq \int_{\partial \Omega} \frac{|y|^{-\beta}}{\left|\nabla G_{\Omega, x}(y)\right|} d \mathcal{H}^{n-1}(y),
$$

which proves the proposition in the present case. The case $\beta=n$ is deduced by a continuity argument from the case $\beta<n$.

For our application in Section 6 the following extension to the level sets of $G_{\Omega, x}$ is crucial. This corollary generalizes Lemma 3 in Lin 25 to the singular case $\beta \neq 0$. 
Corollary 7 Let $n \geq 2, \beta \in[0, n]$ and $\Omega$ be a bounded open smooth subset of $\mathbb{R}^{n}$ with $x \in \Omega$. Then all level sets $A_{r}$ of $G_{\Omega, x}$

$$
A_{r}=\left\{y \in \bar{\Omega}: G_{\Omega, x}(y)>-\frac{1}{\omega_{n-1}^{1 /(n-1)}} \log r\right\}, \quad r \in(0,1]
$$

satisfy the inequality

$$
\omega_{n-1}^{\frac{n}{n-1}} I_{\Omega}^{n-\beta}(x) \leq \frac{1}{r^{n-\beta}} \int_{\partial A_{r}} \frac{|x-y|^{-\beta}}{\left|\nabla G_{\Omega, x}(y)\right|} d \mathcal{H}^{n-1}(y)
$$

Remark 8 It can be shown that the inequality tends to an equality when $r \rightarrow 0$, but this is not required for the proof of Theorem 1 This follows from tha fact that $\lim _{y \rightarrow x}|y-x|\left|\nabla H_{\Omega, x}(y)\right|=0$ (see (1.2) in [21]) and therefore

$$
\left|\nabla G_{\Omega, x}(y)\right|=\frac{1}{\omega_{n-1}^{1 /(n-1)}|x-y|}(1+o(1)) \quad \text { as } y \rightarrow x .
$$

The proof is then similar to that of [25] Lemma 1 (d) and Lemma 3, where it seems that it has been assumed that $\nabla H_{\Omega, x}$ is bounded near $x$. The boundedness of $\nabla H_{\Omega, x}$ has been conjectured in [21] Remark 1.4, but we are not aware whether this has been proven.

Proof We can assume without loss of generality that $x=0 \in \Omega$. Apply Proposition 6 to the set $\Omega=A_{r}$. Note that $0 \in A_{r}$ for all $r \in(0,1]$,

$$
G_{A_{r}, 0}(y)=G_{\Omega, 0}(y)+\frac{1}{\omega_{n-1}^{1 /(n-1)}} \log r \quad \text { and } \quad H_{A_{r}, 0}(y)=H_{\Omega, 0}(y)-\frac{1}{\omega_{n-1}^{1 /(n-1)}} \log r .
$$

In particular this implies that $\nabla G_{A_{r}, 0}=\nabla G_{\Omega, 0}$ and $I_{A_{r}}(0)=r I_{\Omega}(0)$. This proves (14).

\section{Some Preliminary Results}

We first note that it is sufficient to work with non-negative smooth maximizing sequences. More precisely we have the following lemma, which we will use in Section 7 in a crucial way.

Lemma 9 Let $\left\{u_{i}\right\} \subset \mathcal{B}_{1}(\Omega)$ be a sequence such that the limit $\lim _{i \rightarrow \infty} F_{\Omega}\left(u_{i}\right)$ exists. Then there exists a sequence $\left\{w_{i}\right\} \subset \mathcal{B}_{1}(\Omega) \cap C_{c}^{\infty}(\Omega)$ such that

$$
\liminf _{i \rightarrow \infty} F_{\Omega}\left(w_{i}\right) \geq \lim _{i \rightarrow \infty} F_{\Omega}\left(u_{i}\right) .
$$

Moreover, if $u_{i}$ concentrates at $x_{0} \in \bar{\Omega}$, then also $w_{i}$ concentrates at $x_{0}$. In particular maximizing sequences for $F_{\Omega}^{\text {sup }}$ and $F_{\Omega}^{\delta}\left(x_{0}\right)$ can always be assumed to be smooth and non-negative.

Proof The proof is exactly the same as in the case $n=2$, see Lemma 4 in [11. 
Lemma 10 (compactness in interior) Let $0<\eta<1$ and suppose $\left\{u_{i}\right\} \subset W_{0}^{1, n}(\Omega)$ is such that

$$
\limsup _{i \rightarrow \infty}\left\|\nabla u_{i}\right\|_{L^{n}} \leq \eta \quad \text { and } \quad u_{i} \rightarrow u \text { in } W^{1, n}(\Omega)
$$

for some $u \in W^{1, n}(\Omega)$. Then for some subsequence

$$
\frac{e^{\alpha u_{i}^{\frac{n}{n-1}}}}{|x|^{\beta}} \rightarrow \frac{e^{\alpha u^{\frac{n}{n-1}}}}{|x|^{\beta}} \quad \text { in } L^{1}(\Omega)
$$

and in particular $\lim _{i \rightarrow \infty} F_{\Omega}\left(u_{i}\right)=F_{\Omega}(u)$.

Proof The idea of the proof is to apply Vitali convergence theorem. We can assume that, up to a subsequence, that $u_{i} \rightarrow u$ almost everywhere in $\Omega$ and that

$$
\left\|\nabla u_{i}\right\|_{L^{n}} \leq \theta=\frac{1+\eta}{2}<1 \quad \forall i \in \mathbb{N}
$$

We can therefore define $v_{i}=u_{i} / \theta \in \mathcal{B}_{1}(\Omega)$, which satisfies $\left\|\nabla v_{i}\right\|_{L^{n}} \leq 1$ for all $i$. Moreover let us define $\bar{\alpha}=\alpha \theta^{n /(n-1)}<\alpha$, such that

$$
\frac{\bar{\alpha}}{\alpha_{n}}+\frac{\beta}{n}<1
$$

Let $E \subset \Omega$ be an arbitrary measurable set. We use Hölder inequality with exponents $r$ and $s$, where

$$
r=\frac{\alpha_{n}}{\bar{\alpha}}>1 \quad \text { and } \quad \frac{1}{s}=1-\frac{1}{r}>\frac{\beta}{n}
$$

to obtain that

$$
\int_{E} \frac{e^{\alpha u_{i}^{\frac{n}{n-1}}}}{|x|^{\beta}}=\int_{E} \frac{e^{\bar{\alpha} v_{i}^{\frac{n}{n-1}}}}{|x|^{\beta}} \leq\left(\int_{E} e^{\alpha_{n} v_{i}^{\frac{n}{n-1}}}\right)^{\frac{1}{r}}\left(\int_{E} \frac{1}{|x|^{\beta s}}\right)^{\frac{1}{s}} .
$$

Let $\epsilon>0$ be given. In view of the Moser-Trudinger inequality and using that $1 /|x|^{\beta s} \in L^{1}(\Omega)$, we obtain that for any $\epsilon>0$ there exists a $\delta>0$ such that

$$
\int_{E} \frac{e^{\alpha u_{i}^{\frac{n}{n-1}}}}{|x|^{\beta}} \leq \epsilon \quad \forall|E| \leq \delta \text { and } i \in \mathbb{N} .
$$

This shows that the sequence $e^{\alpha u_{i}^{n /(n-1)}} /|x|^{\beta}$ is equi-integrable and the Vitali convergence theorem yields convergence in $L^{1}(\Omega)$. This proves the lemma.

The proof of the next theorem can be found in Lions [24, Theorem I.6.

Theorem 11 (Concentration-Compactness Alternative) Let $\left\{u_{i}\right\} \subset \mathcal{B}_{1}(\Omega)$. Then there is a subsequence and $u \in W_{0}^{1, n}(\Omega)$ with $u_{i} \rightarrow u$ in $W^{1, n}(\Omega)$, such that either

(a) $\left\{u_{i}\right\}$ concentrates at a point $x \in \bar{\Omega}$,

or

(b) the following convergence holds true

$$
\lim _{i \rightarrow \infty} F_{\Omega}\left(u_{i}\right)=F_{\Omega}(u) .
$$


The proof of the next two propositions is the same as in the 2-dimensional case, see [11].

Proposition 12 Let $\beta>0,\left\{u_{i}\right\} \subset \mathcal{B}_{1}(\Omega)$ and suppose that $u_{i}$ concentrates at $x_{0} \in \bar{\Omega}$, where $x_{0} \neq 0$. Then one has that, for some subsequence, $u_{i} \rightarrow 0$ in $W^{1, n}(\Omega)$ and

$$
\lim _{i \rightarrow \infty} F_{\Omega}\left(u_{i}\right)=F_{\Omega}(0)=0 .
$$

In particular $F_{\Omega}^{\delta}\left(x_{0}\right)=0$

Remark 13 However, if $\alpha / \alpha_{n}+\beta / n=1$ then $F_{\Omega}^{\delta}(0)>0$. To see this assume that $\epsilon>0$ is such that $B_{\epsilon}(0) \subset \Omega$ and define

$$
u_{i}(x)=\left\{\begin{aligned}
i & \text { if } 0 \leq|x| \leq \epsilon e^{-\omega_{n-1}^{1 /(n-1)} i^{n /(n-1)}} \\
-\frac{1}{\omega_{n-1}^{1 /(n-1)} i^{1 /(n-1)}} \log \left(\frac{|x|}{\epsilon}\right) & \text { if } \epsilon e^{-\omega_{n-1}^{1 /(n-1)} i^{n /(n-1)}} \leq|x| \leq \epsilon \\
0 & \text { if }|x| \geq \epsilon .
\end{aligned}\right.
$$

One verifies by explicit calculation that $\left\{u_{i}\right\} \subset \mathcal{B}_{1}(\Omega)$ and it concentrates at 0 . Let us show that $\liminf _{i \rightarrow \infty} F_{\Omega}\left(u_{i}\right)>0$. First we make the estimate

$$
F_{\Omega}\left(u_{i}\right)=\int_{\Omega} \frac{e^{\alpha u_{i}^{\frac{n}{n-1}}}-1}{|x|^{\beta}} \geq \int_{B_{a_{i}}(0)} \frac{e^{\alpha u_{i}^{\frac{n}{n-1}}}-1}{|x|^{\beta}}, \quad \text { where } \quad a_{i}=\epsilon e^{-\omega_{n-1}^{\frac{1}{n-1}} i^{\frac{n}{n-1}}} .
$$

To conclude it is sufficient to show that

$$
\lim _{i \rightarrow \infty} \int_{B_{a_{i}}(0)} \frac{e^{\alpha u_{i}^{\frac{n}{n-1}}}-1}{|x|^{\beta}}=\omega_{n-1} \epsilon^{n-\beta}>0 .
$$

We can use that $|x|^{-\beta}$ is integrable and hence

$$
\lim _{i \rightarrow \infty} \int_{B_{a_{i}}(0)}|x|^{-\beta}=0
$$

One calculates that

$$
\int_{B_{a_{i}}(0)} \frac{e^{\alpha u_{i}^{\frac{n}{n-1}}}}{|x|^{\beta}}=\omega_{n-1} \epsilon^{n-\beta} e^{i \frac{n}{n-1}\left(\alpha-(n-\beta) \omega_{n-1}^{\frac{1}{n-1}}\right)}=\omega_{n-1} \epsilon^{n-\beta} \quad \text { if } \frac{\alpha}{\alpha_{n}}+\frac{\beta}{n}=1 .
$$

This shows that $F_{\Omega}^{\delta}(0)>0$.

We first prove Theorem 1 for some simple cases, which is the content of the next proposition. The proof is exactly the same as in the 2-dimensional case, see [11.

Proposition 14 There exists $u \in \mathcal{B}_{1}(\Omega)$ such that $F_{\Omega}(u)=F_{\Omega}^{\text {sup }}$ in the following cases:

$$
\text { (i) } 0 \notin \bar{\Omega} \quad \text { or } \quad \text { (ii) } \frac{\alpha}{\alpha_{n}}+\frac{\beta}{n}<1 \text {. }
$$




\section{The Case $\Omega=B_{1}(0)$.}

In this section we deal with the case where $\Omega$ is the unit ball. The following lemma is essentailly due to Adimurthi-Sandeep [2]. There one can find a proof, which is similar to the 2-dimensional case, see also [11.

Lemma 15 Let $0<a<\infty$, and $u$ be radial function on $B_{1}$. Define

$$
T_{a}(u)(x)=a^{\frac{n-1}{n}} u\left(|x|^{\frac{1}{a}}\right) .
$$

Then $T_{a}$ satisfies that

$$
T_{a}: W_{0, \text { rad }}^{1, n}\left(B_{1}\right) \rightarrow W_{0, \text { rad }}^{1, n}\left(B_{1}\right) .
$$

$T_{a}$ is invertible with $\left(T_{a}\right)^{-1}=T_{1 / a}$ and it satisfies

$$
\left\|\nabla\left(T_{a}(u)\right)\right\|_{L^{n}}=\|\nabla u\|_{L^{n}}, \quad \forall u \in W_{0, \text { rad }}^{1, n}\left(B_{1}\right) .
$$

Moreover if $a=1-\frac{\beta}{n}$, then

$$
F_{B_{1}}(u)=\frac{1}{a} J_{B_{1}}\left(T_{a}(u)\right), \quad \forall u \in W_{0, \text { rad }}^{1, n}\left(B_{1}\right) .
$$

The following corollary follows easily from Lemma 15 .

Corollary 16 Let $a=1-\beta / n$. Then the following identities hold true

$$
\sup _{u \in W_{0, r a d}^{1, n}\left(B_{1}\right) \cap \mathcal{B}_{1}\left(B_{1}\right)} F_{B_{1}}(u)=\frac{1}{a} \sup _{u \in W_{0, r a d}^{1, n}\left(B_{1}\right) \cap \mathcal{B}_{1}\left(B_{1}\right)} J_{B_{1}}(u),
$$

and

$$
F_{B_{1}}^{\text {sup }}=\frac{1}{a} J_{B_{1}}^{\text {sup }} .
$$

Proof The first equality follows directly from Lemma 15. By Schwarz symmetrization, the two equalities of the corollary are equivalent.

One of the crucial ingredients of the proof is the following result of Carleson and Chang [8. Essential is the strict inequality in the following theorem. The second equality is an immediate consequence of the properties of Schwarz symmetrization.

Theorem 17 (Carleson-Chang) The following strict inequality holds true

$$
J_{B_{1}, \text { rad } \searrow}^{\delta}(0)<J_{B_{1}, \text { rad } \searrow}^{\text {sup }}=J_{B_{1}}^{\text {sup }} .
$$

Remark 18 The result in Carleson and Chang is acutally more precise, stating that

$$
e^{1+\frac{1}{2}+\cdots+\frac{1}{n-1}}\left|B_{1}\right|=\sup _{x \in B_{1}} J_{B_{1}, \text { rad } \searrow}^{\delta}(x)<J_{B_{1}, \text { rad } \searrow}^{\text {sup }},
$$

but for our purpose we only need an estimate for the concentration level at 0 . 
From Lemma 15 and Theorem 17 we easily deduce the following proposition.

Lemma 19 Let $\left\{u_{i}\right\} \subset \mathcal{B}_{1}\left(B_{1}\right)$ be a sequence which concentrates at 0 . If $\left\{u_{i}^{*}\right\}$ also concentrates at 0 , then the following strict inequality holds true

$$
\limsup _{i \rightarrow \infty} F_{B_{1}}\left(u_{i}\right)<F_{B_{1}}^{\text {sup }} .
$$

Proof The proof is identical to the 2-dimensional case, see [1], with the only difference that one sets $a=1-\beta / n$.

A consequence of Lemma 19 is the following theorem, stating that the supremum of $F_{B_{1}}$ is attained. The proof is here also identical to the 2-dimensional case.

Theorem 20 The following strict inequality holds

$$
F_{B_{1}}^{\delta}(0)<F_{B_{1}}^{\text {sup }} .
$$

In particular there exists $u \in \mathcal{B}_{1}\left(B_{1}\right)$ such that $F_{B_{1}}^{\text {sup }}=F_{B_{1}}(u)$.

\section{Ball to Domain Construction}

In view of Proposition 14, it remains to prove Theorem 1 for general domain with $0 \in \Omega$, when $\alpha / \alpha_{n}+\beta / n=1$, and we can also take $\beta>0$. Hence from now on we always assume that we are in this case. In addition, we assume in this section and Section 7 that $0 \in \Omega$. The ball to domain construction is given by the following defnition: for $v \in W_{0, r a d}^{1, n}\left(B_{1}\right)$ and $x \in \Omega$, define $P_{x}(v)=u: \Omega \backslash\{x\} \rightarrow \mathbb{R}$ by

$$
P_{x}(v)(y)=v\left(e^{-\omega_{n-1}^{\frac{1}{n-1}} G_{\Omega, x}(y)}\right)=v\left(\left(G_{B_{1}, 0}\right)^{-1}\left(G_{\Omega, x}(y)\right),\right.
$$

where, by abuse of notation, we have identified $v$ and $G_{B_{1}, 0}$ with the corresponding radial function, for instance:

$$
G_{B_{1}, 0}(z)=-\frac{1}{\omega_{n-1}^{1 /(n-1)}} \log z, \quad \text { if } z \in(0,1] .
$$

The main result of this section is the following theorem.

Theorem 21 Assume $\Omega \subset \mathbb{R}^{n}$ is a bounded open smooth set with $0 \in \Omega$. For any $v \in W_{0, \text { rad }}^{1, n}\left(B_{1}\right) \cap$ $\mathcal{B}_{1}\left(B_{1}\right)$ define $u=P_{0}(v)$. Then $u \in \mathcal{B}_{1}(\Omega)$ and it satisfies

$$
F_{\Omega}(u) \geq I_{\Omega}(0)^{n-\beta} F_{B_{1}}(v) .
$$

In particular the following inequality holds true

$$
F_{\Omega}^{\text {sup }} \geq I_{\Omega}(0)^{n-\beta} F_{B_{1}}^{\text {sup }} .
$$

Moreover if $\left\{v_{i}\right\} \subset W_{0, \text { rad }}^{1, n}\left(B_{1}\right)$ concentrates at 0 , then $u_{i}=P_{0}\left(v_{i}\right)$ concentrates at 0 .

The following lemma holds true for any domain, whether containing the origin or not. So we state this general version, although we will use it with $x=0$. 
Lemma 22 Let $x \in \Omega$ and let $v \in W_{0, \text { rad }}^{1, n}\left(B_{1}\right)$. Then $P_{x}(v) \in W_{0}^{1, n}(\Omega)$ and in particular

$$
\left\|\nabla\left(P_{x}(v)\right)\right\|_{L^{n}(\Omega)}=\|\nabla v\|_{L^{n}\left(B_{1}\right)} .
$$

Moreover if $\left\{v_{i}\right\} \subset W_{0, \text { rad }}^{1, n}\left(B_{1}\right)$ concentrates at 0 , then $P_{x}\left(v_{i}\right)$ concentrates at $x$.

Proof Step 1. We write $G=G_{\Omega, x}$. Let $h$ be defined by $h(y)=e^{-\omega_{n-1}^{1 /(n-1)} G(y)}$ and hence $u(y)=$ $v(h(y))$. In particular

$$
\nabla u(y)=v^{\prime}(h(y)) \nabla h(y) .
$$

Note that, since $G \geq 0$ in $\Omega$ we get that if $y \in h^{-1}(\{t\}) \cap \Omega$, then $t \in[0,1]$. Thus the coarea formula gives that

$$
\begin{aligned}
\int_{\Omega}|\nabla u|^{n} & =\int_{\Omega}\left|v^{\prime}(h(y))\right|^{n}|\nabla h(y)|^{n-1}|\nabla h(y)| d y \\
& =\int_{0}^{1}\left[\int_{h^{-1}(\{t\}) \cap \Omega}\left|v^{\prime}(h(y))\right|^{n}|\nabla h(y)|^{n-1} d \mathcal{H}^{n-1}(y)\right] d t .
\end{aligned}
$$

Using that $|\nabla h(y)|=\omega_{n-1}^{1 /(n-1)} h(y)|\nabla G(y)|$, gives

$$
\int_{\Omega}|\nabla u|^{n}=\int_{0}^{1} \omega_{n-1} t^{n-1}\left|v^{\prime}(t)\right|^{n}\left[\int_{h^{-1}(\{t\}) \cap \Omega}|\nabla G(y)|^{n-1} d \mathcal{H}^{n-1}(y)\right] d t .
$$

Note that $h^{-1}(\{t\}) \cap \Omega$ is a level set of $G$. Thus we obtain from Proposition 4 (b) that

$$
\int_{h^{-1}(\{t\}) \cap \Omega}|\nabla G(y)|^{n-1} d \mathcal{H}^{n-1}(y)=1 \quad \forall t \in(0,1),
$$

which implies that

$$
\int_{\Omega}|\nabla u|^{n}=\int_{0}^{1}\left|v^{\prime}(t)\right|^{n} \omega_{n-1} t^{n-1} d t=\int_{B_{1}}|\nabla v|^{n}
$$

This proves (16).

Step 2. Let us now assume that $\left\{v_{i}\right\}$ concentrates at 0 and let $\epsilon>0$ be given. We know from Proposition 4 (e), that for some $M>0$ big enough $\{G>M\} \subset B_{\epsilon}(x)$. Thus we obtain exactly as in Step 1 that

$$
\int_{\Omega \backslash B_{\epsilon}(x)}\left|\nabla u_{i}\right|^{n} \leq \int_{\{G \leq M\}}\left|\nabla u_{i}\right|^{n}=\int_{e^{-\omega_{n-1}^{1 /(n-1)} M}}^{1}\left|v_{i}^{\prime}(t)\right|^{n} \omega_{n-1} t^{n-1} d t .
$$

The right hand side goes to 0 , since $v_{i}$ concentrates. This proves that $u_{i}$ concentrates too.

We are now able to prove the main theorem.

Proof (Theorem 21). We abbreviate again $G=G_{\Omega, 0}$. From Lemma 22 we know that $u \in \mathcal{B}_{1}(\Omega)$. Using the coarea formula we get

$$
\begin{aligned}
F_{\Omega}(u) & =\int_{\Omega} \frac{\left(e^{\alpha u^{n /(n-1)}}-1\right)}{|y|^{\beta}} \frac{|\nabla G(y)|}{|\nabla G(y)|} d y=\int_{0}^{\infty}\left[\int_{G^{-1}(\{t\}) \cap \Omega} \frac{\left(e^{\alpha u^{n /(n-1)}}-1\right)}{|y|^{\beta}|\nabla G(y)|} d \mathcal{H}^{n-1}(y)\right] d t \\
& =\int_{0}^{\infty}\left(e^{\alpha v^{\frac{n}{n-1}}\left(e^{-\omega_{n-1}^{1 /(n-1)} t}\right)}-1\right)\left[\int_{G^{-1}(\{t\}) \cap \Omega} \frac{1}{|y|^{\beta}|\nabla G(y)|} d \mathcal{H}^{n-1}(y)\right] d t .
\end{aligned}
$$


We now use Corollary 7 and set

$$
r(t)=e^{-\omega_{n-1}^{1 /(n-1)} t}
$$

to obtain

$$
\begin{aligned}
F_{\Omega}(u) & \geq I_{\Omega}(0)^{n-\beta} \int_{0}^{\infty} \frac{e^{\alpha v^{\frac{n}{n-1}}(r(t))}-1}{r(t)^{\beta}} \omega_{n-1} \omega_{n-1}^{\frac{1}{n-1}}(r(t))^{n} d t \\
& =-I_{\Omega}(0)^{n-\beta} \int_{0}^{\infty} \frac{e^{\alpha v^{\frac{n}{n-1}}}(r(t))}{r(t)^{\beta}} \omega_{n-1}(r(t))^{n-1} r^{\prime}(t) d t \\
& =I_{\Omega}(0)^{n-\beta} \int_{0}^{1} \frac{e^{\alpha v^{\frac{n}{n-1}}(r)}-1}{r^{\beta}} \omega_{n-1} r^{n-1} d r=I_{\Omega}(0)^{n-\beta} F_{B_{1}}(v) .
\end{aligned}
$$

This proves the first claim of the theorem. The statement about the concentration follows directly from Lemma 22

\section{Domain to Ball Construction}

The aim of this section is to prove the inequality $F_{\Omega}^{\delta}(0) \leq I_{\Omega}(0)^{n-\beta} F_{B_{1}}^{\delta}(0)$. The main difficulty compared to the 2-dimensional case is that we have to deal with the $n$-Laplace equation, which becomes a nonlinear partial differential equation with weaker regularity properties. We summarized the results on $n$-harmonic functions that we need in an Appendix. Recall that we assume $0 \in \Omega$.

Theorem 23 (Concentration Formula) Suppose $\Omega$ contains the origin. Then the following formula holds

$$
F_{\Omega}^{\delta}(0)=I_{\Omega}(0)^{n-\beta} F_{B_{1}}^{\delta}(0) .
$$

The proof of this result will be a consequence of the following proposition, which allows to construct a concentrating sequence in the ball from a given concentrating sequence in $\Omega$.

Proposition 24 Let $\left\{u_{i}\right\} \subset \mathcal{B}_{1}(\Omega) \cap C^{\infty}(\Omega)$ be a sequence which concentrates at 0 and is a maximizing sequence for $F_{\Omega}^{\delta}(0)$. Then there exists a sequence $\left\{v_{i}\right\} \subset W_{0, \text { rad }}^{1, n}\left(B_{1}\right) \cap \mathcal{B}_{1}\left(B_{1}\right)$ concentrating at 0 , such that

$$
F_{\Omega}^{\delta}(0)=\lim _{i \rightarrow \infty} F_{\Omega}\left(u_{i}\right) \leq I_{\Omega}^{n-\beta}(0) \liminf _{i \rightarrow \infty} F_{B_{1}}\left(v_{i}\right) .
$$

Proof (Theorem 23). From Lemma 9 and Proposition 24 we immediately obtain that

$$
F_{\Omega}^{\delta}(0) \leq I_{\Omega}^{n-\beta}(0) F_{B_{1}}^{\delta}(0) .
$$

The reverse inequality follows from Theorem 21.

The proof of Proposition 24 is long and technical. We split it into several intermediate steps. To make the presentation less cumbersome, we assume in what follows that $0 \in \Omega$. However, we actually need this, and the fact that concentration occurs at 0, only in Step 6 in the proof of Lemma 30. The proof of the next two Lemmas is identical to the 2-dimensional case, see [11. 
Lemma 25 Suppose $\left\{u_{i}\right\} \subset \mathcal{B}_{1}(\Omega)$ concentrates at $x_{0} \in \Omega$ and let $\left\{r_{i}\right\} \subset \mathbb{R}$ be such that $r_{i}>0$ for all $i$ and $\lim _{i \rightarrow \infty} r_{i}=0$. Then there exists a subsequence $u_{j_{i}}$ such that

$$
\lim _{i \rightarrow \infty} F_{\Omega}\left(u_{i}\right)=\lim _{i \rightarrow \infty} \int_{\Omega} \frac{e^{\alpha u_{i}^{\frac{n}{n-1}}}-1}{|x|^{\beta}} d x=\lim _{i \rightarrow \infty} \int_{B_{2 r_{i}}\left(x_{0}\right)} \frac{e^{\alpha u_{j_{i}}^{\frac{n}{n-1}}}-1}{|x|^{\beta}} d x .
$$

Moreover any subsequence of $u_{j_{i}}$ will also satisfy the above equality.

Lemma 26 Suppose $\left\{u_{i}\right\}$ is a sequence of measurable non-negative functions such that $u_{i} \rightarrow 0$ almost everywhere in $\Omega$. Let $\left\{s_{i}\right\} \subset \mathbb{R}$ be a bounded sequence. Then

$$
\lim _{i \rightarrow \infty} \int_{\left\{u_{i} \leq s_{i}\right\}} \frac{e^{\alpha u_{i}^{\frac{n}{n-1}}}-1}{|x|^{\beta}} d x=0 .
$$

Lemma 27 Suppose $\left\{u_{i}\right\} \subset \mathcal{B}_{1}(\Omega) \cap C^{\infty}(\Omega)$ concentrates at $0 \in \Omega$ and satisfies

$$
\lim _{i \rightarrow \infty} F_{\Omega}\left(u_{i}\right)=F_{\Omega}^{\delta}(0) .
$$

Then for any $r>0$ there exists $j \in \mathbb{N}$ and $k_{j} \in[1,2]$ such that

$$
\left\{u_{j} \geq k_{j}\right\} \cap B_{r}(0) \neq \emptyset
$$

and all connected components $A$ of $\left\{u_{j} \geq k_{j}\right\}$ will have the property:

$$
\text { If } A \cap B_{r}(0) \neq \emptyset \quad \text { then } A \subset B_{2 r}(0) \text {. }
$$

Moreover A has smooth boundary.

Proof It is sufficient to prove that there exists $j \in \mathbb{N}$ such that (18) is satisfied for $k_{j}=2$ and (19) holds for the connected components of $\left\{u_{j} \geq 1\right\}$, i.e. $k_{j}=1$. This implies that (18) and (19) also hold for any $k \in[0,1]$, and hence, using Sard's theorem, one can choose $k_{j} \in[1,2]$ appropriately such that $A$ has smooth boundary in addition.

First note that for all $m \in \mathbb{N}$ there exists a $j \geq m$ such that (18) must hold. If this is not the case, then Lemma 25 and Lemma 26 imply that

$$
\lim _{i \rightarrow \infty} F_{\Omega}\left(u_{i}\right) \leq \lim _{i \rightarrow \infty} \int_{B_{r}} \frac{e^{\alpha u_{i}^{\frac{n}{n-1}}}-1}{|x|^{\beta}} \leq \lim _{i \rightarrow \infty} \int_{\left\{u_{i} \leq 2\right\}} \frac{e^{\alpha u_{i}^{\frac{n}{n-1}}}-1}{|x|^{\beta}}=0,
$$

which is a contradiction to (17) (Recall that $F_{\Omega}^{\delta}(0)>0$, see Remark 13).

Suppose now that (19) does not hold. We show that this leads to a contradiction, using a capacity argument in dimension $n-1$ (following the idea in [18] Equation (2.12) page 15). In that case there exists for all $j \in \mathbb{N}$ a connected component $D_{j}$ of $\left\{u_{j} \geq 1\right\}$ and $a, b \in \Omega$ such that

$$
a \in D_{j} \cap B_{r} \quad \text { and } \quad b \in D_{j} \cap \Omega \backslash B_{2 r} .
$$

For what follows we fix $j$ and omit the explicit dependence on $j$ (Note that $a$ and $b$ depend on $j$ ). Without loss of generality we can assume, by rotating the domain, that $b=\left(b_{1}, 0\right)$ and $b_{1} \geq 2 r$. 
Therefore, since $D_{j}$ is connected, for all $x_{1} \in[r, 2 r]$ there exists a $X^{\prime}=\left(x_{2}, \cdots, x_{n}\right)=X^{\prime}\left(x_{1}\right) \in$ $\mathbb{R}^{n-1}$ such that $x=\left(x_{1}, X^{\prime}\left(x_{1}\right)\right) \in D_{j}$. In particular $u_{j}(x) \geq 1$. Since $\Omega$ is bounded, there exists an $M>0$, which is independent of the rotation of the domain (and hence of $j$ ), such that $\Omega \subset B_{M}(0)$. In particular this implies that

$$
\left|X^{\prime}\left(x_{1}\right)\right| \leq M \quad \text { for all } x_{1} \in[r, 2 r] .
$$

Let us extend $u_{j}$ by zero in $\mathbb{R}^{n} \backslash \Omega$. Denote by

$$
B_{R}^{\prime}\left(y^{\prime}\right) \text { ball of radius } R \text { in } \mathbb{R}^{n-1} \text { centered at } y^{\prime} \in \mathbb{R}^{n-1} \quad \text { and } \quad \nabla^{\prime} u=\left(\frac{\partial u}{\partial x_{2}}, \ldots, \frac{\partial u}{\partial x_{n}}\right) .
$$

With this notation, using (20), we have $u_{j}\left(x_{1}, y\right)=0$ for $y$ outside of $B_{2 M}^{\prime}\left(X^{\prime}\left(x_{1}\right)\right)$ for all $x_{1} \in$ $(r, 2 r)$. Moreover $u_{j}\left(x_{1}, X^{\prime}\left(x_{1}\right)\right) \geq 1$. Using now the properties of $n$-capacity in $n-1$ dimension, see for instance [19] Example 2.12 pages 35-36,

$$
\begin{aligned}
\int_{B_{2 M}^{\prime}\left(X^{\prime}\left(x_{1}\right)\right)}\left|\nabla^{\prime} u_{j}\left(x_{1}, y^{\prime}\right)\right|^{n} d y^{\prime} & \geq \operatorname{cap}_{n}\left(\left\{X^{\prime}\left(x_{1}\right)\right\}, B_{2 M}^{\prime}\left(X^{\prime}\left(x_{1}\right)\right)\right) \\
& =\operatorname{cap}_{n}\left(\{0\}, B_{2 M}^{\prime}(0)\right)=c(n, M)>0, \quad \text { for all } x_{1} \in(r, 2 r),
\end{aligned}
$$

for some positive constant $c(n, M)$ depending only on $n$ and $M$. Hence, using also that $\Omega$ intersected with any plane where first coordinate equals $x_{1}$ is containded in $\left\{x_{1}\right\} \times B_{M}^{\prime}(0)$, we get

$$
\begin{aligned}
\int_{\Omega \backslash B_{r}(0)}\left|\nabla u_{j}\right|^{n} & \geq \int_{\Omega \backslash B_{r}(0)}\left|\nabla^{\prime} u_{j}\right|^{n} \geq \int_{r}^{2 r} \int_{\Omega \cap\left\{y_{1}=x_{1}\right\}}\left|\nabla^{\prime} u_{j}\left(x_{1}, y^{\prime}\right)\right|^{n} d y^{\prime} d x_{1} \\
& =\int_{r}^{2 r} \int_{B_{M}^{\prime}(0)}\left|\nabla^{\prime} u_{j}\left(x_{1}, y^{\prime}\right)\right|^{n} d y^{\prime} d x_{1}=\int_{r}^{2 r} \int_{B_{2 M}^{\prime}\left(X^{\prime}\left(x_{1}\right)\right)}\left|\nabla^{\prime} u_{j}\left(x_{1}, y^{\prime}\right)\right|^{n} d y^{\prime} d x_{1} \\
& \geq c(n, M) r .
\end{aligned}
$$

This implies that

$$
r c(n, M) \leq \int_{\Omega \backslash B_{r}}\left|\nabla u_{j}\right|^{n} .
$$

But this cannot hold true for all $j$, since $u_{j}$ concentrates at 0 .

The next lemma is about the first modification of the sequence $\left\{u_{i}\right\}$ given in Proposition 24 .

Lemma 28 Let $\left\{u_{i}\right\} \subset \mathcal{B}_{1}(\Omega) \cap C^{\infty}(\Omega)$ be a sequence which concentrates at $0 \in \Omega$ and satisfies

$$
\lim _{i \rightarrow \infty} F_{\Omega}\left(u_{i}\right)=F_{\Omega}^{\delta}(0) .
$$

Then there exists a sequence $\left\{v_{i}\right\} \subset \mathcal{B}_{1}(\Omega)$ and sequences $r_{i}>0$, with $r_{i} \rightarrow 0$ and $\left\{k_{i}\right\} \in[1,2]$ such that

$$
\left\{v_{i} \geq k_{i}\right\} \subset B_{2 r_{i}}, \quad \Delta_{n} v_{i}=0 \quad \text { in }\left\{v_{i}<k_{i}\right\}
$$


Moreover $v_{i}$ has the properties: there exist a sequence $\left\{\lambda_{i}\right\} \subset \mathbb{R}, \lambda_{i}>0$ such that

$$
\begin{aligned}
& \text { (i) } \lim _{i \rightarrow \infty} \lambda_{i}=\infty \\
& \text { (ii) } \lim _{i \rightarrow \infty} v_{i}(y)=0 \quad \text { for all } y \text { in } \Omega \backslash\{0\} \\
& \text { (iii) } \lambda_{i} v_{i} \rightarrow G_{\Omega, 0} \quad \text { in } C_{l o c}^{1}(\Omega \backslash\{0\}) \\
& \text { (iv) } \lim _{i \rightarrow \infty} F_{\Omega}\left(v_{i}\right)=F_{\Omega}^{\delta}(0) .
\end{aligned}
$$

Proof Step 1. Take a sequence of positive real numbers $r_{i}$ such that $\lim _{i \rightarrow \infty} r_{i}=0$ and choose a subsequence of $u_{i}$, using Lemma 25, such that

$$
F_{\Omega}^{\delta}(0)=\lim _{i \rightarrow \infty} F_{\Omega}\left(u_{i}\right)=\lim _{i \rightarrow \infty} \int_{B_{r_{i}}} \frac{e^{\alpha u_{i}^{\frac{n}{n-1}}}-1}{|x|^{\beta}} .
$$

Choosing again a subsequence we can assume by Lemma 27 that there exist $k_{i} \in[1,2]$ such that all connected components $A$ of $\left\{u_{i} \geq k_{i}\right\}$ which intersect $B_{r_{i}}$ are contained in $B_{2 r_{i}}$. We define $A_{i}$ as the union of all such $A$. We also know from Lemma 27 that $A_{i}$ is not empty. Let $w_{i} \in W^{1, n}\left(\Omega \backslash \bar{A}_{i}\right)$ be the solution of, see Theorem 32 .

$$
\left\{\begin{array}{c}
\Delta_{n} w_{i}=0 \quad \text { in } \Omega \backslash \bar{A}_{i} \\
w_{i}=0 \quad \text { on } \partial \Omega, \quad w_{i}=k_{i} \quad \text { on } \partial A_{i} .
\end{array}\right.
$$

We now define $v_{i} \in W_{0}^{1, n}(\Omega)$ as

$$
v_{i}= \begin{cases}u_{i} & \text { in } A_{i} \\ w_{i} & \text { in } \Omega \backslash A_{i} .\end{cases}
$$

Since $n$-harmonic functions minimize the $n$-Dirichlet integral we have $\left\|\nabla v_{i}\right\|_{L^{n}} \leq\left\|\nabla u_{i}\right\|_{L^{n}}$. Thus we have constructed a sequence which has the properties: (we have used Theorem 35] in the second property)

$$
\left\{v_{i} \geq k_{i}\right\} \subset B_{2 r_{i}}, \quad \Delta_{n} v_{i}=0 \quad \text { in }\left\{v_{i}<k_{i}\right\} \quad \text { and } \quad\left\|\nabla v_{i}\right\|_{L^{n}} \leq 1 .
$$

Step 2. We will show in this Step that for all $y \in \Omega \backslash\{0\}$ we have $v_{i}(y)>0$ for all $i$ large enough and $\lim _{i \rightarrow \infty} v_{i}(y)=0$. The fact that $v_{i}(y)>0$ follows from the maximum principle Theorem 35, Since $\Omega$ is bounded there exists $M>0$ such that $\bar{\Omega} \subset B_{M}$. Define $W_{i}=B_{M} \backslash \overline{B_{2 r_{i}}}$ and let $\psi_{i}$ be the solution of

$$
\left\{\begin{array}{c}
\Delta_{n} \psi_{i}=0 \quad \text { in } W_{i} \\
\psi_{i}=2 \text { on } \partial B_{2 r_{i}} \text { and } \psi_{i}=0 \text { on } \partial B_{M} .
\end{array}\right.
$$

The function $\psi_{i}$ can be given explicitly:

$$
\psi_{i}=\frac{2}{\log \left(\frac{2 r_{i}}{M}\right)} \log \left(\frac{|x|}{M}\right) .
$$

Recall that $k_{i} \in[1,2]$ and note that

$$
\begin{aligned}
& \psi_{i}>0 \text { and } v_{i}=0 \quad \text { on } \partial \Omega, \\
& \psi_{i}=2 \text { and } v_{i}<k_{i} \leq 2 \quad \text { on } \partial B_{2 r_{i}},
\end{aligned}
$$


and thus $\psi_{i}-v_{i}>0$ on $\partial W_{i}$. Since $v_{i}$ is also harmonic in $W_{i}$ the comparison principle (Theorem (33) implies that $v_{i} \leq \psi_{i}$ in $W_{i}$. For $i$ big enough $y \in W_{i}$ and the claim of Step 2 follows from the fact that $\lim _{i \rightarrow \infty} \psi_{i}(y)=0$.

Step 3. Choose $y \in \Omega \backslash\{0\}$ and define $\lambda_{i}$ by

$$
\lambda_{i}=\frac{G_{\Omega, 0}(y)}{v_{i}(y)} \Leftrightarrow \lambda_{i} v_{i}(y)=G_{\Omega, 0}(y)
$$

In view of Step 2 this is well defined, $\lambda_{i}>0$ and

$$
\lim _{i \rightarrow \infty} \lambda_{i}=\infty
$$

Let $y \in K_{1} \subset \Omega \backslash\{0\}$ be a compact set. Choose another compact set $K_{2}$, such that $K_{1} \subset \subset K_{2} \subset$ $\Omega \backslash\{0\}$. Applying Harnack inequality (Theorem 34) on $K_{2}$ we get that there exist $c_{1}, c_{2}>0$, such that

$$
c_{1}\left|G_{\Omega, 0}(y)\right| \leq\left|\lambda_{i} v_{i}(x)\right| \leq c_{2}\left|G_{\Omega, 0}(y)\right|, \quad \forall x \in K_{2} \text { and } \forall i \text { large enough. }
$$

Hence the sequence $\lambda_{i} v_{i}$ is uniformly bounded in the $C^{0}\left(K_{2}\right)$ norm. It follows from Theorem 36 that

$$
\lambda_{i} v_{i} \quad \text { is uniformly bounded in the } C^{1, \alpha}\left(K_{1}\right) \text { norm }
$$

for some $0<\alpha$. Using the compact embedding $C^{1, \alpha}\left(K_{1}\right) \hookrightarrow C^{1}\left(K_{1}\right)$ we obtain that there exists $g \in C^{1}\left(K_{1}\right)$ and a subsequence $v_{i}$ with

$$
g_{i}:=\lambda_{i} v_{i} \rightarrow g \quad \text { in } C^{1}\left(K_{1}\right) .
$$

It follows from (22) and Corollary 38 that $g=G_{\Omega, 0}$, once we have shown that $g=0$ on $\partial \Omega$. We prove this claim in the next step.

Step 4. We show now that $g=0$ on $\partial \Omega$. Define $\Omega_{\epsilon}$ as

$$
\Omega_{\epsilon}=\{x \in \Omega: 0<\operatorname{dist}(x, \partial \Omega)<\epsilon\},
$$

where $\epsilon$ will be chosen later small enough, and

$$
\partial \Omega_{\epsilon}=\partial \Omega \cup \Gamma_{\epsilon} \quad \text { where } \quad \Gamma_{\epsilon}=\{x \in \Omega: \operatorname{dist}(x, \partial \Omega)=\epsilon\} .
$$

Step 4.1. We claim that $g_{i}$ are uniformly bounded on $\Omega_{\epsilon}$. Note that $g_{i}=0$ on $\partial \Omega$. So for small enough $\epsilon$ it follows from Lemma 40 and Remark 41)(ii) (as in the proof of Proposition 39) that

$$
\left\|g_{i}\right\|_{L^{\infty}\left(\Omega_{\epsilon}\right)} \leq C\left(\Omega_{\epsilon}\right)\left\|g_{i}\right\|_{L^{n}\left(\Omega_{\epsilon}\right)} .
$$

We now fix such an $\epsilon>0$ for which (25) holds and we can also assume that $\Omega_{\epsilon}$ is a smooth set. It follows from (24) that there exists $\Lambda_{1}=\Lambda_{1}\left(K_{1}, K_{2}\right)$ (chosing $K_{1}$ such that $\Gamma_{\epsilon} \subset K_{1}$ ), such that

$$
\left\|g_{i}\right\|_{C^{1, \alpha}\left(\Gamma_{\epsilon}\right)} \leq \Lambda_{1} \quad \text { for all } i \text { big enough, }
$$

and hence also for some $\Lambda_{2}>0$

$$
\left\|g_{i}\right\|_{W^{1-\frac{1}{n}, n}\left(\Gamma_{\epsilon}\right)} \leq \Lambda_{2} \quad \text { for all } i \text { big enough. }
$$


Chose now a bounded right inverse $T$ of the trace operator on $\partial \Omega_{\epsilon}$ as

$$
T: W^{1-\frac{1}{n}}\left(\partial \Omega_{\epsilon}\right) \rightarrow W^{1, n}\left(\Omega_{\epsilon}\right)
$$

and apply it to $g_{i}$ restricted to $\partial \Omega_{\epsilon}$. Hence there exists $h_{i} \in W^{1, n}\left(\Omega_{\epsilon}\right), h_{i}=T\left(g_{i}\right)$ such that

$$
h_{i}=g_{i} \quad \text { on } \partial \Omega_{\epsilon}
$$

and

$$
\left\|h_{i}\right\|_{W^{1, n}\left(\Omega_{\epsilon}\right)} \leq C_{1}\left(\Omega_{\epsilon}\right)\left\|g_{i}\right\|_{W^{1-\frac{1}{n}, n}\left(\partial \Omega_{\epsilon}\right)} \leq C_{1}\left(\Omega_{\epsilon}\right) \Lambda_{2} .
$$

As in the proof of Proposition 39] since $\Delta_{n} g_{i}=0$ in $\Omega_{\epsilon}$ for $i$ big enough,

$$
\begin{aligned}
\left\|g_{i}-h_{i}\right\|_{L^{n}\left(\Omega_{\epsilon}\right)} \leq C_{2}\left(\Omega_{\epsilon}\right)\left\|\nabla g_{i}-\nabla h_{i}\right\|_{L^{n}\left(\Omega_{\epsilon}\right)} \leq & C_{2}\left(\left\|\nabla g_{i}\right\|_{L^{n}\left(\Omega_{\epsilon}\right)}+\left\|\nabla h_{i}\right\|_{L^{n}\left(\Omega_{\epsilon}\right)}\right) \\
& \leq 2 C_{2}\left\|\nabla h_{i}\right\|_{L^{n}\left(\Omega_{\epsilon}\right)} \leq 2 C_{1} C_{2} \Lambda_{2} .
\end{aligned}
$$

Since the $h_{i}$ are also uniformly bounded by (26) it follows the $g_{i}$ are uniformly bounded in the $L^{\infty}\left(\Omega_{\epsilon}\right)$ norm. This shows Step 4.1.

Step 4.2. We now conclude that $g=0$ on $\partial \Omega$. Fix some $a>0$ so that $B_{a}(0) \subset \subset \Omega$ and define $\Omega_{a}=\Omega \backslash \overline{B_{a}}(0)$. Note that $g_{i}$ is uniformly bounded on $\partial B_{a}(0)$ in the $C^{1, \alpha}$ norm, using again (24), i.e. for some $\Lambda_{3}>0$ we have

$$
\left\|g_{i}\right\|_{C^{1, \alpha}\left(\partial B_{a}(0)\right)} \leq \Lambda_{3} .
$$

On the compact set $\Omega \backslash\left(B_{a}(0) \cup \Omega_{\epsilon}\right) g_{i}$ is uniformly bounded by (23). Thus, together with Step 4.1 this shows that there exists a constant $M_{0}$ independent of $i$ such that

$$
\left\|g_{i}\right\|_{L^{\infty}\left(\Omega_{a}\right)} \leq M_{0}
$$

Note that

$$
\Delta_{n} g_{i}=0 \quad \text { in } \Omega_{a} .
$$

So it follows from Theorem 32 that

$$
\left\|g_{i}\right\|_{C^{1, \alpha}\left(\overline{\Omega_{a}}\right)} \leq C\left(M_{0}, \Lambda_{3}\right)
$$

It follows that for some subsequence $g_{i} \rightarrow g$ in $C^{1}\left(\overline{\Omega_{a}}\right)$ from which it follows that $g=0$ on $\partial \Omega$.

Step 5. It remains to prove (iv). Recall that $v_{i} \leq k_{i}$ in $\Omega \backslash A_{i}$. We therefore obtain, using Lemma 26 twice and the definition of $A_{i}$ that

$$
\begin{aligned}
& \lim _{i \rightarrow \infty} \int_{\Omega} \frac{e^{\alpha v_{i}^{\frac{n}{n-1}}}-1}{|x|^{\beta}}=\lim _{i \rightarrow \infty} \int_{A_{i}} \frac{e^{\alpha u_{i}^{\frac{n}{n-1}}}-1}{|x|^{\beta}} \geq \lim _{i \rightarrow \infty} \int_{A_{i} \cap B_{r_{i}}} \frac{e^{\alpha u_{i}^{\frac{n}{n-1}}}-1}{|x|^{\beta}} \\
= & \lim _{i \rightarrow \infty} \int_{\left\{u_{i} \geq k_{i}\right\} \cap B_{r_{i}}} \frac{e^{\alpha u_{i}^{\frac{n}{n-1}}}-1}{|x|^{\beta}}=\lim _{i \rightarrow \infty} \int_{B_{r_{i}}} \frac{e^{\alpha u_{i}^{n-1}}-1}{|x|^{\beta}}=F_{\Omega}^{\delta}(0),
\end{aligned}
$$

where we have used (21) in the last equality.

The next lemma is about the second modification of the sequence $\left\{u_{i}\right\}$ given in Proposition 24. following the first modification given by Lemma 28 . 
Lemma 29 Let $\left\{u_{i}\right\} \subset W_{0}^{1, n}(\Omega)$ be a sequence and $\lambda_{i}$ a sequence in $\mathbb{R}$ such that $\lambda_{i} \rightarrow \infty$,

$$
\lambda_{i} u_{i} \rightarrow G_{\Omega, 0} \quad \text { in } C_{l o c}^{0}(\Omega \backslash\{0\}) \quad \text { and } \quad \Delta_{n} u_{i}=0 \text { in }\left\{u_{i}<1\right\} .
$$

Then there exists a subsequence $\lambda_{i_{l}}$ and a sequence $\left\{v_{l}\right\} \subset W_{0}^{1, n}(\Omega)$ such that the following properties hold true:

(a) $\lambda_{i_{l}} \geq l$

(b) The sets $\left\{v_{l} \geq l / \lambda_{i_{l}}\right\}$ are approximately small balls at 0 as $l \rightarrow \infty$.

(c) $v_{l}(x) \rightarrow 0$ as $l \rightarrow \infty$ for every $x$ in $\Omega \backslash\{0\}$.

(d) For every $l$

$$
\int_{\Omega}\left|\nabla v_{l}\right|^{n} \leq \int_{\Omega}\left|\nabla u_{i_{l}}\right|^{n}
$$

(e) The inequality $v_{l} \geq u_{i_{l}}$ holds in $\Omega$. In particular $F_{\Omega}\left(v_{l}\right) \geq F_{\Omega}\left(u_{i_{l}}\right)$.

Proof The proof is exactly the same as in the 2-dimensional case, since one can use the strong maximum principle, Theorem [35, for $n$-harmonic functions.

After having modified the sequence $\left\{u_{i}\right\}$ given in Proposition 24 in the two previous lemmas, we finally construct the appropriate corresponding sequence $\left\{v_{i}\right\} \subset W_{0, \text { rad }}^{1, n}\left(B_{1}\right)$. This is contained in the following lemma.

Lemma 30 Let $\left\{u_{i}\right\} \subset W_{0}^{1, n}(\Omega)$ and $\left\{s_{i}\right\} \subset \mathbb{R}$ be sequences with the following properties:

$$
s_{i} \leq 1 \quad \forall i \in \mathbb{N},
$$

the sets $\left\{u_{i} \geq s_{i}\right\}$ are approximately small balls at 0 as $i \rightarrow \infty$ and moreover suppose that pointwise $u_{i}(x) \rightarrow 0$ for all $x \in \Omega \backslash\{0\}$. Then there exists a sequence $\left\{v_{i}\right\} \subset W_{0, \text { rad }}^{1, n}\left(B_{1}\right)$ such that for all $i$

$$
\left\|\nabla v_{i}\right\|_{L^{n}\left(B_{1}\right)} \leq\left\|\nabla u_{i}\right\|_{L^{n}(\Omega)}
$$

and, assuming that the left hand side limit exists,

$$
\lim _{i \rightarrow \infty} F_{\Omega}\left(u_{i}\right) \leq I_{\Omega}(0)^{n-\beta} \lim \inf _{i \rightarrow \infty} F_{B_{1}}\left(v_{i}\right) .
$$

Moreover $v_{i}(x) \rightarrow 0$ for all $x \in B_{1} \backslash\{0\}$ and if $v_{i}$ concentrates at some $x_{0} \in B_{1}$, then $x_{0}=0$.

Proof Throughout this proof $G=G_{\Omega, 0}$ shall denote the $n$-Green's function of $\Omega$ with singularity at 0 . Recall that by assumption there exists real positive numbers $\rho_{i}$ and $\epsilon_{i}$ such that for $i \rightarrow \infty$

$$
\rho_{i} \rightarrow 0 \quad \text { and } \quad \frac{\epsilon_{i}}{\rho_{i}} \rightarrow 0,
$$

satisfying for all $i$ the following inclusion

$$
B_{\rho_{i}-\epsilon_{i}} \subset\left\{u_{i} \geq s_{i}\right\} \subset B_{\rho_{i}+\epsilon_{i}} .
$$

Step 1. Let us define $\lambda_{i}$, implicitly, by the following equation:

$$
\rho_{i}=I_{\Omega}(0) e^{-\omega_{n-1}^{1 /(n-1)} \lambda_{i}},
$$


that is

$$
\lambda_{i}=-\frac{1}{\omega_{n-1}^{1 /(n-1)}} \log \left(\frac{\rho_{i}}{I_{\Omega}(0)}\right) .
$$

Note that $\lambda_{i} \rightarrow \infty$ as $i \rightarrow \infty$. We claim that there exists $t_{i} \geq \lambda_{i}$ such that

$$
\lim _{i \rightarrow \infty}\left(t_{i}-\lambda_{i}\right)=0
$$

and

$$
\left\{G \geq t_{i}\right\} \subset\left\{u_{i} \geq s_{i}\right\} .
$$

To show this we use Proposition 4 (e), which states that if $t_{i} \geq 0$ is given such that $t_{i} \rightarrow \infty$, then there exists $\sigma_{i} \geq 0$ such that

$$
\lim _{i \rightarrow \infty} \frac{\sigma_{i}}{\tau_{i}}=0
$$

and

$$
B_{\tau_{i}-\sigma_{i}} \subset\left\{G \geq t_{i}\right\} \subset B_{\tau_{i}+\sigma_{i}}
$$

where $\tau_{i}=I_{\Omega}(0) e^{-\omega_{n-1}^{1 /(n-1)} t_{i}}$. In view of (28) it is therefore sufficient to choose $t_{i}$ such that

$$
\tau_{i}+\sigma_{i}=\rho_{i}-\epsilon_{i}
$$

It remains to show that with this choice (30) is also satisfied. Using (29) and solving the previous equation for $t_{i}$ explicitly gives that

$$
t_{i}=\lambda_{i}-\frac{1}{\omega_{n-1}^{1 /(n-1)}} \log \left(1-\frac{\epsilon_{i}+\sigma_{i}}{\rho_{i}}\right) .
$$

Since we know from (27) that $\epsilon_{i} / \rho_{i} \rightarrow 0$, it is sufficient to show that $\sigma_{i} / \rho_{i} \rightarrow 0$. We obtain from (32) that

$$
\frac{\sigma_{i}}{\tau_{i}}=\frac{\sigma_{i}}{\rho_{i}-\epsilon_{i}-\sigma_{i}}=\frac{\sigma_{i}}{\rho_{i}\left(1-\frac{\epsilon_{i}}{\rho_{i}}-\frac{\sigma_{i}}{\rho_{i}}\right)} .
$$

Solving this equation for $\left(\sigma_{i} / \rho_{i}\right)$ and using that $\epsilon_{i} / \rho_{i} \rightarrow 0$ and $\sigma_{i} / \tau_{i} \rightarrow 0$ shows that also $\left(\sigma_{i} / \rho_{i}\right) \rightarrow$ 0 . This proves (30).

Step 2. In this step we will show that

$$
\int_{\left\{u_{i}<1\right\}}\left|\nabla u_{i}\right|^{n} \geq \frac{s_{i}^{n}}{t_{i}^{n-1}} .
$$

Let us denote

$$
U=\left\{u_{i} \geq s_{i}\right\} \quad \text { and } \quad V=\left\{G \geq t_{i}\right\} .
$$

From Step 1 we know that $V \subset U$ and since $u_{i}=0$ on $\partial \Omega$ we also have that $\bar{U} \subset \Omega$. Let $h_{i} \in W^{1, n}(\Omega \backslash V)$ be the unique solution of the problem

$$
\begin{gathered}
\Delta_{n} h_{i}=0 \quad \text { in } \Omega \backslash V \\
h_{i}=0 \quad \text { on } \partial \Omega \quad \text { and } \quad h_{i}=1 \quad \text { on } \partial V .
\end{gathered}
$$


We see that this is satisfied precisely by $h_{i}=G / t_{i}$. Let us define $w_{i} \in W^{1, n}(\Omega \backslash V)$ by

$$
w_{i}= \begin{cases}\frac{u_{i}}{s_{i}} & \text { in } \Omega \backslash U \\ 1 & \text { in } \bar{U} \backslash V .\end{cases}
$$

Note that $w_{i}$ has the same boundary values as $h_{i}$ on the boundary of $\Omega \backslash V$. Since $h_{i}$ is the unique minimizer of the functional

$$
J(h)=\int_{\Omega \backslash V}|\nabla h|^{n}
$$

among all functions with these fixed boundary values, we get that

$$
\int_{\Omega \backslash V}\left|\nabla h_{i}\right|^{n} \leq \int_{\Omega \backslash V}\left|\nabla w_{i}\right|^{n}=\int_{\Omega \backslash U}\left|\nabla w_{i}\right|^{n}=\int_{\left\{u_{i}<s_{i}\right\}}\left|\nabla u_{i}\right|^{n} .
$$

From Proposition 4 (a) we know that

$$
\int_{\Omega \backslash V}\left|\nabla h_{i}\right|^{n}=\int_{\left\{G<t_{i}\right\}}\left|\nabla\left(\frac{G}{t_{i}}\right)\right|^{n}=\frac{1}{t_{i}^{n-1}} .
$$

Setting this into the previous inequality proves (33).

Step 3. In this step we will define $v_{i} \in W_{0, \text { rad }}^{1, n}\left(B_{1}\right)$. Let $\Omega^{*}=B_{R}$ be the symmetrized domain and $u_{i}^{*} \in W_{0, \text { rad }}^{1, n}\left(B_{R}\right)$ be the radially decreasing symmetric rearrangement of $u_{i}$. Then there exists $0<a_{i}<R$ such that

$$
\left\{u_{i}^{*} \geq s_{i}\right\}=B_{a_{i}} .
$$

Moreover define $0<\delta_{i}<1$ by

$$
\delta_{i}=e^{-\omega_{n-1}^{1 /(n-1)} t_{i}}
$$

At last we can define $v_{i}$ as

$$
v_{i}(x)= \begin{cases}-\frac{s_{i}}{\omega_{n-1}^{1 /(n-1)} t_{i}} \log (|x|) & \text { if } x \geq \delta_{i} \\ u_{i}^{*}\left(\frac{a_{i}}{\delta_{i}} x\right) & \text { if } x \leq \delta_{i} .\end{cases}
$$

Note that $v_{i}$ belongs indeed to $W^{1, n}\left(B_{1}\right)$ since

$$
u_{i}^{*}\left(a_{i}\right)=s_{i}=-\frac{s_{i}}{\omega_{n-1}^{1 /(n-1)} t_{i}} \log \left(\delta_{i}\right) .
$$

Step 4. In this Step we will show that $\left\|\nabla v_{i}\right\|_{L^{n}\left(B_{1}\right)} \leq\left\|\nabla u_{i}\right\|_{L^{n}(\Omega)}$. Let us denote

$$
A_{i}=\int_{B_{1} \backslash B_{\delta_{i}}}\left|\nabla v_{i}\right|^{n} \quad \text { and } D_{i}=\int_{B_{\delta_{i}}}\left|\nabla v_{i}\right|^{n} .
$$

A direct calculation gives that

$$
A_{i}=\frac{\omega_{n-1} s_{i}^{n}}{\omega_{n-1}^{n /(n-1)} t_{i}^{n}} \int_{\delta_{i}}^{1} \frac{1}{r} d r=\frac{s_{i}^{n}}{t_{i}^{n-1}}
$$


Using a change of variables and Proposition 2 (ii) gives that

$$
D_{i}=\int_{B_{a_{i}}}\left|\nabla u_{i}^{*}\right|^{n}=\int_{\left\{u_{i}^{*} \geq s_{i}\right\}}\left|\nabla u_{i}^{*}\right|^{n} \leq \int_{\left\{u_{i} \geq s_{i}\right\}}\left|\nabla u_{i}\right|^{n} .
$$

Finally we get that, using (33), that

$$
\int_{B_{1}}\left|\nabla v_{i}\right|^{n}=D_{i}+A_{i} \leq \int_{\Omega}\left|\nabla u_{i}\right|^{n}-\int_{\left\{u_{i}<s_{i}\right\}}\left|\nabla u_{i}\right|^{n}+\frac{s_{i}^{n}}{t_{i}^{n-1}} \leq \int_{\Omega}\left|\nabla u_{i}\right|^{n} .
$$

Step 5. In this step we show that

$$
\lim _{i \rightarrow \infty} \frac{a_{i}}{\delta_{i}}=I_{\Omega}(0)
$$

Using the fact that $\left|\left\{u_{i}^{*} \geq s_{i}\right\}\right|=\left|\left\{u_{i} \geq s_{i}\right\}\right|$, we get from (34) and the hypthesis (28) we get that $\rho_{i}-\epsilon_{i} \leq a_{i} \leq \rho_{i}+\epsilon_{i}$. From this equation we obtain that

$$
\frac{\rho_{i}}{\delta_{i}}\left(1-\frac{\epsilon_{i}}{\rho_{i}}\right) \leq \frac{a_{i}}{\delta_{i}} \leq \frac{\rho_{i}}{\delta_{i}}\left(1+\frac{\epsilon_{i}}{\rho_{i}}\right) .
$$

From the hypothesis (27) we know that $\epsilon_{i} / \rho_{i} \rightarrow 0$. It is therefore sufficient to calculate the limit of $\rho_{i} / \delta_{i}$. In view the definitions of $\rho_{i}, \delta_{i}$ and (30) this is indeed equal to

$$
\lim _{i \rightarrow \infty} \frac{\rho_{i}}{\delta_{i}}=\lim _{i \rightarrow \infty} I_{\Omega}(0) e^{\omega_{n-1}^{1 /(n-1)}\left(t_{i}-\lambda_{i}\right)}=I_{\Omega}(0),
$$

which proves the statement of this step.

Step 6 (equality of functional limit). Let us first show that both $u_{i}$ and $v_{i}$ converge to zero almost everywhere. For $u_{i}$ this holds true by hypothesis. So let $x \in B_{1} \backslash\{0\}$ be given and note that for all $i$ big enough

$$
x \geq e^{-\omega_{n-1}^{1 /(n-1)} \sqrt{t_{i}}} \geq e^{-\omega_{n-1}^{1 /(n-1)} t_{i}}=\delta_{i} .
$$

Therefore we obtain from the definition of $v_{i}$ that

$$
v_{i}(x) \leq-\frac{s_{i}}{\omega_{n-1}^{1 /(n-1)} t_{i}} \log \left(e^{-\omega_{n-1}^{1 /(n-1)} \sqrt{t_{i}}}\right)=\frac{s_{i}}{\sqrt{t_{i}}} \rightarrow 0,
$$

which shows the claim also for $v_{i}$. In view of lemma 26 it is therefore sufficient to show that

$$
\lim _{i \rightarrow \infty} \int_{\left\{u_{i} \geq s_{i}\right\}} \frac{e^{\alpha u_{i}^{n /(n-1)}}-1}{|x|^{\beta}}=I_{\Omega}^{n-\beta}(0) \liminf _{i \rightarrow \infty} \int_{\left\{v_{i} \geq s_{i}\right\}} \frac{e^{\alpha v_{i}^{n /(n-1)}}-1}{|x|^{\beta}} .
$$

From Proposition 2 (i) and the properties of symmetrization we get that for every $i$

$$
\int_{\left\{u_{i} \geq s_{i}\right\}} \frac{e^{\alpha u_{i}^{n /(n-1)}}-1}{|x|^{\beta}} \leq \int_{\left\{u_{i}^{*} \geq s_{i}\right\}} \frac{e^{\alpha\left(u_{i}^{*}\right)^{n /(n-1)}}-1}{\left(|x|^{\beta}\right)^{*}}=\int_{B_{a_{i}}} \frac{e^{\alpha\left(u_{i}^{*}\right)^{n /(n-1)}}-1}{\left(|x|^{\beta}\right)^{*}} .
$$


Note that if $\beta=0$ then the inequality can actally be replaced by an equality (see Kesavan, page 14, equation (1.3.2)). For $i$ big enough $B_{a_{i}} \subset \Omega$, and then $\left(|x|^{\beta}\right)^{*}=|x|^{\beta}$ for all $x \in B_{a_{i}}$. Making the substitution $x=\left(a_{i} / \delta_{i}\right) y$ gives

$$
\int_{\left\{u_{i} \geq s_{i}\right\}} \frac{e^{\alpha u_{i}^{n /(n-1)}}-1}{|x|^{\beta}} \leq\left(\frac{a_{i}}{\delta_{i}}\right)^{n-\beta} \int_{B_{\delta_{i}}} \frac{e^{\alpha v_{i}^{n /(n-1)}}-1}{|y|^{\beta}}=\left(\frac{a_{i}}{\delta_{i}}\right)^{n-\beta} \int_{\left\{v_{i} \geq s_{i}\right\}} \frac{e^{\alpha v_{i}^{n /(n-1)}}-1}{|x|^{\beta}} .
$$

From Step 5 we therefore get that

$$
\lim _{i \rightarrow \infty} \int_{\left\{u_{i} \geq s_{i}\right\}} \frac{e^{\alpha u_{i}^{n /(n-1)}}-1}{|x|^{\beta}} \leq I_{\Omega}(0)^{n-\beta} \liminf _{i \rightarrow \infty} \int_{\left\{v_{i} \geq s_{i}\right\}} \frac{e^{\alpha v_{i}^{n /(n-1)}}-1}{|x|^{\beta}},
$$

which proves (36).

Step 7. The last statement of the lemma ( $v_{i}$ can concentrate only at 0 ) follows from (35), because $\lim _{i \rightarrow \infty} A_{i}=0$ (using that $t_{i} \rightarrow \infty$ ). Thus $v_{i}$ cannot concentrate at any other point than 0 .

We are now able to prove the main proposition of this section.

Proof (Proposition 24). The proof is exactly the same as in the 2-dimensional case, see details in 11: One modifies succesively the given sequence $u_{i}$ using Lemma 28 and Lemma 29 and construct finally a sequence $\left\{v_{i}\right\} \subset W_{0, \text { rad }}^{1, n}\left(B_{1}\right) \cap \mathcal{B}_{1}\left(B_{1}\right)$, using Lemma [30, such that

$$
F_{\Omega}^{\delta}(0) \leq \lim _{i \rightarrow \infty} F_{\Omega}\left(u_{i}\right) \leq I_{\Omega}^{n-\beta}(0) \liminf _{i \rightarrow \infty} F_{B_{1}}\left(v_{i}\right) .
$$

The sequence $\left\{v_{i}\right\}$ has to concentrate at 0 , because it goes to zero almost everywhere. (use last statment of Lemma 30, Theorem 11 and Remark 13).

\section{Proof of the Main Result}

We now prove Theorem 1 We assume that $0 \in \Omega$, the other cases are proven exactly as in the two dimensional setting. For the case $0 \in \partial \Omega$ use [45] Proposition $2.4(3)$, which implies that $I_{\Omega_{m}}(0)$ tends to zero if $\left\{\Omega_{m}\right\}$ is a sequence of sets whose boundary approaches the origin.

Proof Let $\left\{u_{i}\right\}_{i \in \mathbb{N}} \subset \mathcal{B}_{1}(\Omega)$ be a maximizing sequence for $F_{\Omega}$. Then by Theorem 11 for some subsequence, either

$$
\lim _{i \rightarrow \infty} F_{\Omega}\left(u_{i}\right)=F_{\Omega}(u),
$$

or $\left\{u_{i}\right\}$ concentrates at some point $x \in \bar{\Omega}$. In the first case $u$ is an extremal function and we are done. It remains to exclude concentration. Assume, by contradiction, we have concentration at $x$. By Proposition 12 we must have $x=0$ (because we have assumed $\left\{u_{i}\right\}$ is a maximizing sequence and $F_{\Omega}^{\text {sup }} \neq 0$ ). Thus we get

$$
F_{\Omega}^{\mathrm{sup}}=\lim _{i \rightarrow \infty} F_{\Omega}\left(u_{i}\right)=F_{\Omega}^{\delta}(0) .
$$

By Theorem 23 it holds

$$
F_{\Omega}^{\delta}(0)=I_{\Omega}(0)^{n-\beta} F_{B_{1}}^{\delta}(0)
$$

and by Theorem 20

$$
F_{B_{1}}^{\delta}(0)<F_{B_{1}}^{\text {sup }}
$$


Finally Theorem 21 states

$$
F_{B_{1}}^{\text {sup }} I_{\Omega}(0)^{n-\beta} \leq F_{\Omega}^{\text {sup }} .
$$

Combining (37)-(40) gives the contradiction $F_{\Omega}^{\text {sup }}<F_{\Omega}^{\text {sup }}$, and therefore concentration cannot occur.

\section{Appendix: $n$-harmonic functions}

We summarize the results on $n$-harmonic functions that we have used. We only state them under the more restrictive hypothesis that are sufficient for our construction of the $n$-harmonic transplantation and we refer to references for more general versions.

Definition 31 Let $\Omega \subset \mathbb{R}^{n}$ be an open set and let $f \in W^{1, n}(\Omega)$. we say that $f$ is $n$-harmonic, that is

$$
\Delta_{n} f=0 \quad \text { in } \Omega,
$$

if

$$
\int_{\Omega}|\nabla f|^{n-2}\langle\nabla f, \nabla \varphi\rangle=0 \quad \text { for all } \varphi \in C_{c}^{\infty}(\Omega) .
$$

The existence of $W^{1, n}$ solutions to the $n$-Laplace equation follows easily by direct methods of the calculus of variations. The difficult part of the next theorem is the $C^{1, \alpha}$ regularity. For the interior regularity see [15, 38, or [16. Lieberman [22] proves regularity up to the boundary under the assumption that $f$ is bounded. In this paper we only need the regularity result for very special boundary values (constants on different parts of the boundary). In this case the proof is simple, so we have included a proof of the boundedness, see Proposition 39. For a more general version, not assuming the boundedness of $f$, we have not found a satisfactory reference.

Theorem 32 (Existence and Regularity) There exists $0<\alpha<1$, depending only on $n$, with the following property. Let $\Omega \subset \mathbb{R}^{n}$ be a bounded open smooth set, $g \in C^{1, \alpha}(\partial \Omega)$ such that $\|g\|_{C^{1, \alpha}(\partial \Omega)} \leq t_{0}$. Then there exists a unique $f \in W^{1, n}(\Omega)$ such that

$$
\begin{aligned}
\Delta_{n} f & =0 & & \text { in } \Omega \\
f & =g & & \text { on } \partial \Omega .
\end{aligned}
$$

The solution $f$ satisfies

$$
\int_{\Omega}|\nabla f|^{n} \leq \int_{\Omega}|\nabla \varphi|^{n} \quad \forall \varphi \in W^{1, n}(\Omega) \text { with } \varphi=g \quad \text { on } \partial \Omega .
$$

Moreover if $\|f\|_{L^{\infty}}(\Omega) \leq M_{0}$ then $f \in C^{1, \alpha}(\bar{\Omega})$ and there exists a constant $C$ depending on $n, \Omega, M_{0}$ and $t_{0}$ such that

$$
\|f\|_{C^{1, \alpha}(\bar{\Omega})} \leq C\left(n, \Omega, M_{0}, t_{0}\right)
$$

A reference for the next three results is for instance [23] Theorems 2.15, 2.20 and Corollary 2.21. 
Theorem 33 (Comparison Principle) Let $\Omega \subset \mathbb{R}^{n}$ be a bounded open smooth set. Suppose $f, g \in W^{1, n}(\Omega)$ and $\Delta_{n} f=\Delta_{n} g=0$ in $\Omega$. If

$$
f \leq g \quad \text { on } \partial \Omega
$$

then

$$
f \leq g \quad \text { in } \Omega .
$$

Theorem 34 (Harnack Inequality) Let $V$ be a compact set and $U$ open with $V \subset \subset U$. Then there is a constant $C=C(V)$ such that

$$
\sup _{V} u \leq C \inf _{V} u
$$

for all $u \in W^{1, n}(U)$ such that $\Delta_{n} u=0$ in $U$ and $u \geq 0$ in $U$.

Theorem 35 (Strong maximum principle) Let $\Omega$ be a bounded open set with smooth boundary $\partial \Omega$ and $u \in W^{1, n}(\Omega)$. Suppose $\Delta_{n} u=0$ in $\Omega, u$ is not constant and $u \geq 0$. Then the following holds

$$
\inf _{\partial \Omega} f<f(x)<\sup _{\partial \Omega} f \quad \text { for all } x \in \Omega .
$$

The next theorem follows from 38] Theorem 1.

Theorem 36 Let $V$ be a compact set $V \subset \subset U$, where is $U$ open. Then there exists $\alpha \in(0,1)$ and a constant $C>0$ depending on $V, U, n$, and a variable $g_{0}$ such that

$$
\|u\|_{C^{1, \alpha}(V)} \leq C\left(V, U, n, g_{0}\right)
$$

for all $u \in W^{1, n}(U)$ with $\Delta_{n} u=0$ in $U$ and $=\|u\|_{L^{\infty}(U)} \leq g_{0}$.

The next theorem is contained in 34. Theorem 10 and 35] Theorem 3, combined with Theorem 32 This is the generalization of Bocher's theorem (see [5] Theorem 3.9 page 50) to $n$-harmonic functions.

Theorem 37 Let $\Omega \subset \mathbb{R}^{n}$ be a bounded open smooth set with $0 \in \Omega, u \in W_{\text {loc }}^{1, n}(\Omega \backslash\{0\})$ with the properties

$$
\Delta_{n} u=0 \quad \text { in } \Omega \backslash\{0\}
$$

Then

(a) either $u \in L^{\infty}(\Omega)$, in which case $u$ is continuous in $\Omega$. Moreove $u \in W^{1, n}(\Omega)$ and it solves the equation

$$
\Delta_{n} u=0 \quad \text { in } \Omega,
$$

and hence $u \in C_{\text {loc }}^{1, \alpha}(\Omega)$ for some $0<\alpha<1$.

(b) or there exists a constant $k \in \mathbb{R}$ such that

$$
\int_{\Omega}\left\langle|\nabla u|^{n-2} \nabla u, \nabla \varphi\right\rangle=k \varphi(0) \quad \text { for all } \varphi \in C_{c}^{1}(\Omega) .
$$


Corollary 38 Suppose $u \in W^{1, n}(\Omega \backslash\{0\}), \Delta_{n} u=0$ in $\Omega \backslash\{0\}$ and $u=0$ on $\partial \Omega$. Then either $u$ is a constant multiple of the $n$-Green's function, i.e. $u=k G_{\Omega, 0}(y)$ or $u$ vanishes identically.

The next lemma states that the hypothesis of the regularity result of Lieberman 22$]$ is satisfied, that is, the boundedness of $f$ by $M_{0}$ in Proposition 32

Proposition 39 Let $\Omega \subset \mathbb{R}^{n}$ be a bounded opens smooth set such that $\partial \Omega=\bigcup_{i=1}^{L} \Gamma_{i}$ where $\Gamma_{i}$ are the connected smooth components of $\partial \Omega$. Let $k_{i} \in \mathbb{R}, i=1, \ldots, L$ be constants such that $\left|k_{i}\right| \leq k$. Then there exists a constant $M_{0}=M_{0}(\Omega, n, k)$ such that

$$
\|u\|_{L \infty}(\Omega) \leq M_{0}(\Omega, n, k)
$$

for all $u \in W^{1, n}(\Omega)$ satisfying $\Delta_{n} u=0$ in $\Omega$ and $u=k_{i}$ on $\Gamma_{i}$

The following lemma is required for the proof of Proposition 39 and has also been used in Lemma 28

Lemma 40 Let $\Omega, \Gamma_{i}, u$ and $k_{i}$ be as in Proposition [39. Then there exists constants $C=C(\Omega, n)>$ $0, q=q(\Omega, n)>1$ such that for any $x \in \Gamma_{i}$ and $0<r<R$ with $B_{R}(x) \cap \partial \Omega \subset \Gamma_{i}$ (that is the ball of radius $R$ does not intersect other connected parts of the boundary) one has

$$
\left\|\left(u-k_{i}\right)_{+}\right\|_{L^{\infty}\left(B_{r}(x) \cap \Omega\right)} \leq \frac{C}{(R-r)^{q}}\left\|\left(u-k_{i}\right)_{+}\right\|_{L^{n}\left(B_{R}(x) \cap \Omega\right)} .
$$

The same holds true for $\left(u-k_{i}\right)_{-}$instead of $\left(u-k_{i}\right)_{+}$.

Remark 41 (i) The proof of the lemma shows that $1<q<\infty$ can be choosen freely, in which case $C$ depends also on $q$.

(ii) The Lemma is a local result and requires $u$ to be $n$-harmonic only in a neighborhood of the ball $B_{R}(x)$ and constant on $\partial \Omega \cap B_{R}(x)$.

Proof (Proposition 39). By standard interior regularity, see for instance 23] Lemma 3.6, the estimate (42) holds true also for balls $B_{R}(x)$ contained in $\Omega$ (with $k_{i}$ replaced by 0 ). Since $\partial \Omega$ is smooth and compact and its connected components $\Gamma_{i}$ have positive distance between eachother, we can cover the boundary by finitely many balls $B_{r}(x), x \in \partial \Omega$ satisfying the estimate (42). Summing up all these estimates we obtain that there exists a constant $C=C(\Omega)$ such that

$$
\|u\|_{L^{\infty}(\Omega)} \leq C\left(\|u\|_{L^{n}(\Omega)}+k\right) .
$$

Choose a function $g \in W^{1, n}(\Omega)$ such that $g=k_{i}$ on $\Gamma_{i}$ (take for instance a bounded linear extension operator $\left.T: W^{1-1 / n, n}(\partial \Omega) \rightarrow W^{1, n}(\Omega)\right)$ so that

$$
\|g\|_{W^{1, n}}(\Omega) \leq C(\Omega) k .
$$

It follows from Poincaré inequality $(u-g=0$ on $\partial \Omega)$ and using (41) that

$$
\|u-g\|_{L^{n}(\Omega)} \leq C\|\nabla u-\nabla g\|_{L^{n}(\Omega)} \leq C\left(\|\nabla u\|_{L^{n}(\Omega)}+\|\nabla g\|_{L^{n}(\Omega)}\right) \leq 2 C\|\nabla g\|_{L^{n}(\Omega)} \leq 2 C k
$$

From this we obtain the desired estimate for $\|u\|_{L^{n}}$ which we plug into (43) to conclude. 
We now prove Lemma 40, by a standard Moser iteration method.

Proof (Lemma 40) Since the Lemma is a local result and $\Delta_{n}\left(u-k_{i}\right)=0$, we can assume without loss of generality that $k_{i}=0$ and $u=0$ on $\Gamma_{i}$. The estimate (42) is clearly also valid for $\left(u-k_{i}\right)_{-}$ instead of $\left(u-k_{i}\right)_{+}$, by applying a posteriori the Lemma 40 to $-u$.

Step 1. We shall first prove that if $\chi>1$ and $\gamma \geq n$. Then there exists a constant $C=$ $C(\Omega, n, \chi)>0$ such that for any $x \in \Gamma_{i}$ and $0<t<T$ such that $B_{T}(x) \cap \partial \Omega \subset \Gamma_{i}$

$$
\left\|u_{+}\right\|_{L^{\gamma \chi}\left(B_{t}(x) \cap \Omega\right)} \leq\left(\frac{C \gamma}{T-t}\right)^{\frac{n}{\gamma}}\left\|u_{+}\right\|_{L^{\gamma}\left(B_{T}(x) \cap \Omega\right)},
$$

provided the right hand side is finite. We fix and write henceforth for simplicity

$$
B_{T}(x)=B_{T}, \quad B_{t}(x)=B_{t} \quad \text { and } \quad \Omega_{T}=\Omega \cap B_{T}(x), \quad \Omega_{t}=\Omega \cap B_{t}(x)
$$

Step 1.1. By assumption we have that

$$
\int_{\Omega}\left\langle|\nabla u|^{n-2} \nabla u, \nabla \varphi\right\rangle=0 \quad \text { for all } \varphi \in W_{0}^{1, n}(\Omega) .
$$

Let $u_{+}=\max \{0, u\}$. Let $\xi \in C_{c}^{1}\left(B_{T}\right)$. We define for $m \in \mathbb{N}$ and $\beta \geq n-1$

$$
u_{m}(y)=\left\{\begin{aligned}
u_{+}(y) & \text { if } u(y)<m \\
m & \text { if } u(y) \geq m,
\end{aligned} \quad \text { and } \quad \varphi=\xi^{n} u_{m}^{\beta} u \in W_{0}^{1, n}(\Omega)\right.
$$

which we plug into (45). Deriving we obtain

$$
\nabla \varphi=\xi^{n} u_{m}^{\beta} \nabla u+\beta \xi^{n} u_{m}^{\beta-1} u \nabla u_{m}+n \xi^{n-1} u_{m}^{\beta} u \nabla \xi .
$$

This gives that

$$
\int_{\Omega_{T}} \xi^{n} u_{m}^{\beta}|\nabla u|^{n}+\beta \int_{\Omega_{T}} \xi^{n} u_{m}^{\beta-1} u\left\langle\nabla u_{m}, \nabla u\right\rangle|\nabla u|^{n-2}=-n \int_{\Omega_{T}} \xi^{n-1} u_{m}^{\beta} u\langle\nabla \xi, \nabla u\rangle|\nabla u|^{n-2} .
$$

In the second term $\nabla u_{m}$ appears, which is zero if $u \geq m$, and therefore we can replace everywhere $u$ by $u_{m}$ to get

$$
\int_{\Omega_{T}} \xi^{n} u_{m}^{\beta}|\nabla u|^{n}+\beta \int_{\Omega_{T}} \xi^{n} u_{m}^{\beta}\left|\nabla u_{m}\right|^{n}=-n \int_{\Omega_{T}} \xi^{n-1} u_{m}^{\beta} u\langle\nabla \xi, \nabla u\rangle|\nabla u|^{n-2} .
$$

Take $\epsilon>0$, which we will soon chose appropriately. We use the estimate

$$
a b \leq \frac{(n-1) a^{n /(n-1)}}{n}+\frac{b^{n}}{n}
$$

with $a=\xi^{n-1}|\nabla u|^{n-1} u_{m}^{\beta(n-1) / n} \epsilon$ and $b=u_{m}^{\beta / n} u|\nabla \xi| / \epsilon$ to estimate the right side of (46)

$$
-n \int_{\Omega_{T}} \xi^{n-1} u_{m}^{\beta} u\langle\nabla \xi, \nabla u\rangle|\nabla u|^{n-2} \leq(n-1) \int_{\Omega_{T}} \xi^{n} u_{m}^{\beta}|\nabla u|^{n} \epsilon^{\frac{n}{n-1}}+\int_{\Omega_{T}} u_{m}^{\beta} u^{n}|\nabla \xi|^{n} \frac{1}{\epsilon^{n}} .
$$


So for an appropriate choice of $\epsilon=\epsilon(n)$ we obtain a constant $C=C(n)$ such that

$$
\int_{\Omega_{T}} \xi^{n} u_{m}^{\beta}|\nabla u|^{n}+\beta \int_{\Omega_{T}} \xi^{n} u_{m}^{\beta}\left|\nabla u_{m}\right|^{n} \leq C \int_{\Omega_{T}} u_{m}^{\beta} u^{n}|\nabla \xi|^{n} .
$$

We now set

$$
w=u_{m}^{\frac{\beta}{n}} u, \quad \Rightarrow \quad \nabla w=\frac{\beta}{n} u_{m}^{\frac{\beta}{n}-1} \nabla u_{m} u+u_{m}^{\frac{\beta}{n}} \nabla u .
$$

Recall that if in any product $\nabla u_{m}$ appears, then $u=u_{m}$ in that product, so we obtain

$$
\begin{aligned}
|\nabla w|^{n} \leq & \left(\frac{\beta}{n}\left|u_{m}^{\frac{\beta}{n}}\right|\left|\nabla u_{m}\right|+u_{m}^{\frac{\beta}{n}}|\nabla u|\right)^{n} \leq 2^{n-1}\left(\left(\frac{\beta}{n}\right)^{n} u_{m}^{\beta}\left|\nabla u_{m}\right|^{n}+u_{m}^{\beta}|\nabla u|^{n}\right) \\
& =2^{n-1}\left(\frac{\beta^{n-1}}{n^{n}} \beta u_{m}^{\beta}\left|\nabla u_{m}\right|^{n}+u_{m}^{\beta}|\nabla u|^{n}\right) \\
& \leq 2^{n-1}\left(1+\frac{\beta^{n-1}}{n^{n}}\right)\left(\beta u_{m}^{\beta}\left|\nabla u_{m}\right|^{n}+u_{m}^{\beta}|\nabla u|^{n}\right)
\end{aligned}
$$

We use this in (47) to obtain that, renaming $2^{n-1} C$ by $C$ (as we will do henceforth)

$$
\int_{\Omega_{T}} \xi^{n}|\nabla w|^{n} \leq C\left(1+\frac{\beta^{n-1}}{n^{n}}\right) \int_{\Omega_{T}} w^{n}|\nabla \xi|^{n}
$$

Now use that

$$
|\nabla(\xi w)|^{n} \leq(|\xi||\nabla w|+|\nabla \xi||w|)^{n} \leq 2^{n-1}\left(|\xi|^{n}|\nabla w|^{n}+|\nabla \xi|^{n}|w|^{n}\right) .
$$

We thus get that

$$
\int_{\Omega_{T}}|\nabla(\xi w)|^{n} \leq C\left(1+\frac{\beta^{n-1}}{n^{n}}\right) \int_{\Omega_{T}} w^{n}|\nabla \xi|^{n},
$$

Note that $\xi w \in W_{0}^{1, n}(\Omega)$ and that $W_{0}^{1, n}(\Omega) \hookrightarrow L^{q}(\Omega)$ for any $1 \leq q<\infty$ by the Sobolev embedding. In particular there exists a constant $C=C(\chi, \Omega, n)$ such that

$$
\left(\int_{\Omega_{T}}|\xi w|^{\chi^{n}}\right)^{\frac{1}{\chi}}=\left(\int_{\Omega}|\xi w|^{\chi^{n}}\right)^{\frac{1}{\chi}} \leq C(\chi, \Omega, n) \int_{\Omega}|\nabla(\xi w)|^{n}=C(\chi, \Omega, n) \int_{\Omega_{T}}|\nabla(\xi w)|^{n} .
$$

So we obtain from (48)

$$
\left(\int_{\Omega_{T}}|\xi w|^{\chi^{n}}\right)^{\frac{1}{\chi}} \leq C\left(1+\frac{\beta^{n-1}}{n^{n}}\right) \int_{\Omega_{T}} w^{n}|\nabla \xi|^{n}
$$

We now choose $\xi$ such that $\xi=1$ in $B_{t}$ and $|\nabla \xi| \leq 2 /(T-t)$. We also assume that $u$ has been extende by zero outside of $\Omega$ (more precisely on the other side of $\Gamma_{i}$ ). Thus, using the definition of $w=u_{m}^{\beta / n} u$ we get

$$
\left(\int_{B_{t}}\left(u_{m}^{\frac{\beta}{n}} u\right)^{\chi n}\right)^{\frac{1}{\chi}} \leq C\left(1+\frac{\beta^{n-1}}{n^{n}}\right) \frac{2^{n}}{(T-t)^{n}} \int_{B_{T}} u_{m}^{\beta} u^{n} \leq C\left(1+\frac{\beta^{n-1}}{n^{n}}\right) \frac{2^{n}}{(T-t)^{n}} \int_{B_{T}} u_{+}^{\beta+n}
$$


We now let $m \rightarrow \infty$ and obtain that

$$
\left(\int_{B_{t}} u_{+}^{\chi(\beta+n)}\right)^{\frac{1}{\chi}} \leq C\left(1+\frac{\beta^{n-1}}{n^{n}}\right) \frac{2^{n}}{(T-t)^{n}} \int_{B_{T}} u_{+}^{\beta+n}
$$

Set now $\gamma=\beta+n$ and we get that

$$
\left(\int_{B_{t}} u_{+}^{\gamma \chi}\right)^{\frac{1}{\chi}} \leq C\left(1+\frac{(\gamma-n)^{n-1}}{n^{n}}\right) \frac{1}{(T-t)^{n}} \int_{B_{T}} u_{+}^{\gamma} \leq C \gamma^{n} \frac{1}{(T-t)^{n}} \int_{B_{T}} u_{+}^{\gamma} .
$$

From this (44) follows.

Step 2. We now use (44) iteratively. Set

$$
t_{j}=r+\frac{R-r}{2^{j}}, \quad \gamma_{j}=\chi^{j} n \quad \text { for } j=0,1,2, \ldots
$$

Note that $t_{0}=R, t_{j}>t_{j+1}$ and $\gamma_{0}=n, \gamma_{j+1}=\chi \gamma_{j}$. Moreover $t_{j}-t_{j+1}=(R-r) / 2^{j+1}$. So it follows from (44) (by induction the right hand side of the next inequality is bounded for each $j$ ) that

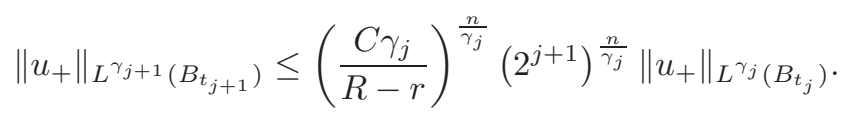

Note that

$$
\begin{aligned}
\left(\frac{C \gamma_{j}}{R-r}\right)^{\frac{n}{\gamma_{j}}}\left(2^{j+1}\right)^{\frac{n}{\gamma_{j}}} & =\left(\frac{C \chi^{j} n}{R-r}\right)^{\frac{1}{\chi^{j}}}\left(2^{j+1}\right)^{\frac{1}{\chi^{j}}}=\left(2 C \chi^{j} n 2^{j}\right)^{\frac{1}{\chi^{j}}}(R-r)^{-\frac{1}{\chi^{j}}} \\
& \leq D^{\frac{j}{\chi^{j}}}(R-r)^{-\frac{1}{\chi^{j}}}
\end{aligned}
$$

for some constant $D=D(\Omega, n, \chi)$. We now set $a_{j}=\left\|u_{+}\right\|_{L^{\gamma_{j}}\left(B_{t_{j}}\right)}$ and notice $a_{0}=\left\|u_{+}\right\|_{L^{n}\left(B_{R}\right)}$. Thus we obtain from (49) that

$$
a_{j+1} \leq D^{\frac{j}{\chi^{j}}}(R-r)^{-\frac{1}{\chi^{j}}} a_{j}
$$

It follows by induction that

$$
a_{j+1} \leq D^{\sum_{l=0}^{j} \frac{l}{\chi^{l}}}(R-r)^{-\sum_{l=0}^{j} \frac{1}{\chi^{l}}} a_{0} .
$$

Notice that for all $j$ we have $\left\|u_{+}\right\|_{L^{n \chi^{j}\left(B_{r}\right)}} \leq a_{j+1}$. The sums

$$
\sum \frac{l}{\chi^{l}} \text { and } \sum \frac{1}{\chi^{l}}
$$

are convergent. So letting $j \rightarrow \infty$ we obtain that

$$
\left\|u_{+}\right\|_{L^{\infty}\left(B_{r}\right)} \leq \frac{C}{(R-r)^{q}}\left\|u_{+}\right\|_{L^{n}\left(B_{R}\right)}
$$

which proves the lemma.

Acknowledgements The first author was supported by Chilean Fondecyt Iniciación grant nr. 11150017 and is member of the Barcelona Graduate School of Mathematics. He also acknowledges 
financial support from the Spanish Ministry of Economy and Competitiveness, through the "María de Maeztu" Programme for Units of Excellence in RD (MDM-2014-0445). The research work of the second author is supported by the Indian "Innovation in Science Pursuit for Inspired Research (INSPIRE)" under the IVR Number: 20140000099 (IFA14-MA43).

We have obtained significant help from A. Adimurthi, who helped us understand and work out the relevant results on the $n$-Laplace equation. We would like to extend our thanks to Sandeep for

fruitful discussions on this problem. Finally, our special thanks goes to Debabrata Karmakar for his generous help.

\section{References}

[1] Adimurthi and Druet O., Blow-up analysis in dimension 2 and a sharp form of Trudinger-Moser inequality. Comm. Partial Differential Equations 29 (2004), no. 1-2, 295-322.

[2] Adimurthi and Sandeep K., A singular Moser-Trudinger embedding and its applications, NoDEA Nonlinear Differential Equations Appl., 13 (2007), no. 5-6, 585-603.

[3] Adimurthi and Tintarev C., On compactness in the Trudinger-Moser inequality. Ann. Sc. Norm. Super. Pisa Cl. Sci. (5) 13 (2014), no. 2, 399-416.

[4] A. Alvino, F. Brock, F. Chiacchio, A. Mercaldo, and M. R. Posteraro, Some isoperimetric inequalities on $\mathbb{R}^{N}$ with respect to weights $|x|^{a}$, J. Math. Anal. Appl., 451 (2017), no. 1, 280-318.

[5] Axler S., Bourdon P., and Ramey W., Harmonic function theory, Second edition, Graduate Texts in Mathematics, 137, Springer-Verlag, New York, 2001.

[6] Beardon and Alan F., The geometry of discrete groups. Graduate Texts in Mathematics, 91. SpringerVerlag, New York, 1983.

[7] Betta M.F., Brock F., Mercaldo A., and Posteraro M.R., A weighted isoperimetric inequality and applications to symmetrization, J. of Inequal. and Appl., 4 (1999), 215-240.

[8] Carleson L. and Chang S.-Y. A., On the existence of an extremal function for an inequality by J. Moser, Bull. Sci. Math., (2) 110 (1986), no. 2, 113-127.

[9] Csató G., An isoperimetric problem with density and the Hardy-Sobolev inequality in $\mathbb{R}^{2}$, Differential Integral Equations, 28, Number 9/10 (2015), 971-988.

[10] Csató G., On the isoperimetric problem with perimeter density $r^{p}$, Commun. on Pure and Appl. Anal. 17 (2018), no. 6, 2729-2749.

[11] Csató G., and Roy P., Extremal functions for the singular Moser-Trudinger inequality in 2 dimensions, Calc. Var. Partial Differential Equations, 54 (2015), no. 2, 2341-2366.

[12] Csató G. and Roy P., The singular Moser-Trudinger inequality on simply connected domains, Comm. Partial Differential Equations, 41 (2016), no. 5, 838-847.

[13] Chen W. X. and Li C., Classification of solutions of some nonlinear elliptic equations, Duke Math. J., 63 (1991), no. 3, 615-622.

[14] Chen W. X., and Li C., What kinds of singular surfaces can admit constant curvature?, Duke Math. J., 78 (1995), no. 2, 437-451.

[15] E. diBenedetto, $C^{1, \alpha}$ local regularity of weak solutions of degenerate elliptic equations, Nonlinear Analyisis, Theory, Methods and Applications, Vol. 7 (1983), 827-850.

[16] Evans L.C., A new proof of local $C^{1, \alpha}$ regularity of solutions of certain degenerate elliptic p.d.e., J. Differential Equations, Volume 45, issue 3 (1982) 356-373.

[17] Flucher M., Extremal functions for the Trudinger-Moser inequality in 2 dimensions, Comment. Math. Helvetici, 67 (1992), 471-497.

[18] Flucher M., Variational problems with concentration, Progress in Nonlinear Differential Equations and their Applications, 36. Birkhäuser Verlag, Basel, 1999. 
[19] Heinonen J., Kilpeläinen T., and Martio O., Non-linear potential theory of degenerate elliptic equations, Oxford Mathematical Monographs. Oxford Science Publications. The Clarendon Press, Oxford University Press, New York, 1993.

[20] Kesavan S., Symmetrization and applications, Series in Analysis, 3. World Scientific Publishing Co. Pte. Ltd., Hackensack, NJ, 2006.

[21] Kichensassamy S., and Veron L., Singular solutions of the p-Laplace equation, Math. Ann., 275 (1986) 599-615.

[22] Gary M. L., Boundary regularity for solutions of degenerate elliptic equations, Nonlinear Analyisis, Theory, Methods and Applications, Vol 12 (1988) 1203-1219.

[23] Lindqvist P., Notes on the p-Laplace equation, Report. University of Jyväskylä Department of Mathematics and Statistics, 102. University of Jyväskylä, Jyväskylä, 2006.

[24] Lions P.-L., The concentration-compactness principle in the calculus of variations. The limit case. I, Rev. Mat. Iberoamericana 1, (1985), no. 1, 145-201.

[25] Lin K.C., Extremal functions for Moser's inequality, Trans. of. Am. Math. Soc., 348 (1996), 26632671.

[26] Lula S., and Mancini G., Extremal Functions for Singular Moser-Trudinger Embeddings, Nonlinear Anal., 156 (2017), 215-248.

[27] Malchiodi A. and Martinazzi L., Critical points of the Moser-Trudinger functional on a disk, J. Eur. Math. Soc. (JEMS), 16 (2014), no. 5, 893-908.

[28] Mancini G., and Martinazzi L., The Moser-Trudinger inequality and its extremals on a disk via energy estimates, arXiv:1608.07169.

[29] Mancini G., and Sandeep K., Moser-Trudinger inequality on conformal discs. Commun. Contemp. Math., 12 (2010), no. 6, 1055-1068.

[30] Ngo Q-A., and Nguyen V. H., An improved Moser-Trudinger inequality involving the first non-zero Neumann eigenvalue with mean value zero in $\mathbb{R}^{2}$, arxiv.org/abs/1702.08883.

[31] Nguyen V. H., A sharp Adams inequality in dimension four and its extremal functions, arxiv.org/abs/1701.08249.

[32] Moser J., A sharp form of an inequality by N. Trudinger, Indiana Univ. Math. J., 20 (1971), no. 11, 1077-1092.

[33] Roy P., Extremal function for Moser-Trudinger type inequality with logarithmic weight, Nonlinear Analysis, 135 (2016), 194-204.

[34] Serrin J., Local behavior of solutions of quasi-linear equations Acta Math., 111 (1964) $247-302$.

[35] Serrin J., Isolated singularities of solutions of quasilinear equations, Acta Math., 113 (1965) 219-240.

[36] Struwe M., Critical points of embeddings of $H_{0}^{1, n}$ into Orlicz spaces, Ann. Inst. H. Poincaré Anal. Non Linaire, 5 (1988), no. 5, 425-464.

[37] Nguyen V. H. and Takahashi F., On a weighted Trudinger-Moser type inequality on the whole space and related maximizing problem, Differential Integral Equations, 31 (2018), no. 11-12, 785-806.

[38] Tolksdorf P., Regularity for a more general class of quasilinear elliptic equations, J. Differential Equations, 51 (1984), no. 1, 126-150.

[39] Trudinger N. S., On embeddings into Orlicz spaces and some applications, J. Math. Mech., 17 (1967), 473-484.

[40] Yang Y., Extremal functions for a sharp Moser-Trudinger inequality. Internat. J. Math., 17 (2006), no. 3, 331-338.

[41] Yang Y., Extremal functions for Moser-Trudinger inequalities on 2-dimensional compact Riemannian manifolds with boundary. Internat. J. Math., 17 (2006), no. 3, 313-330.

[42] Yang Y., Extremal functions for Trudinger-Moser inequalities of Adimurthi-Druet type in dimension two. J. Differential Equations, 258 (2015), no. 9, 3161-3193. 
[43] Yang Y., A Trudinger-Moser inequality on a compact Riemannian surface involving Gaussian curvature. J. Geom. Anal. 26 (2016), no. 4, 2893-2913.

[44] Yang Y., and Zhu B., Blow-up analysis concerning singular Trudinger-Moser inequalities in dimension 2, J. Funct. Anal., 272 (2017), 3347-3374.

[45] Wang W., $N$-capacity, $N$-harmonic radius and $N$-harmonic transplantation, J. Math. Anal. Appl. 327 (2007), no. 1, 155-174. 